ARTICLE

\title{
Improved CRISPR genome editing using small highly active and specific engineered RNA-guided nucleases
}

Moritz J. Schmidt ${ }^{1,4}$, Ashish Gupta ${ }^{1,4}$, Christien Bednarski ${ }^{1,4}$, Stefanie Gehrig-Giannini ${ }^{1}$, Florian Richter ${ }^{1}$, Christian Pitzler ${ }^{1}$, Michael Gamalinda ${ }^{1}$, Christina Galonska ${ }^{1}$, Ryo Takeuchi ${ }^{2}$, Kui Wang ${ }^{2}$, Caroline Reiss ${ }^{2}$, Kerstin Dehne", Michael J. Lukason ${ }^{3}$, Akiko Noma ${ }^{2}$, Cindy Park-Windhol ${ }^{2}$, Mariacarmela Allocca², Albena Kantardzhieva ${ }^{2}$, Shailendra Sane ${ }^{2}$, Karolina Kosakowska², Brian Cafferty², Jan Tebbe ${ }^{1}$, Sarah J. Spencer ${ }^{3}$, Scott Munzer ${ }^{2}$, Christopher J. Cheng ${ }^{2}$, Abraham Scaria ${ }^{2}$, Andrew M. Scharenberg ${ }^{2}$, André Cohnen (1) ${ }^{1,5 凶} \&$ Wayne M. Coco (1) $1,5 \bowtie$

Streptococcus pyogenes (Spy) Cas9 has potential as a component of gene therapeutics for incurable diseases. One of its limitations is its large size, which impedes its formulation and delivery in therapeutic applications. Smaller Cas9s are an alternative, but lack robust activity or specificity and frequently recognize longer PAMs. Here, we investigated four uncharacterized, smaller Cas9s and found three employing a "GG" dinucleotide PAM similar to SpyCas9. Protein engineering generated synthetic RNA-guided nucleases (sRGNs) with editing efficiencies and specificities exceeding even SpyCas9 in vitro and in human cell lines on disease-relevant targets. sRGN mRNA lipid nanoparticles displayed manufacturing advantages and high in vivo editing efficiency in the mouse liver. Finally, sRGNs, but not SpyCas9, could be packaged into all-in-one AAV particles with a gRNA and effected robust in vivo editing of non-human primate (NHP) retina photoreceptors. Human gene therapy efforts are expected to benefit from these improved alternatives to existing CRISPR nucleases.

\footnotetext{
${ }^{1}$ Bayer AG, Leverkusen, Germany. ${ }^{2}$ Casebia Therapeutics LLC, Cambridge, MA, USA. ${ }^{3}$ CRISPR Therapeutics INC, Cambridge, MA, USA. ${ }^{4}$ These authors contributed equally: Moritz J. Schmidt, Ashish Gupta, Christien Bednarski. ${ }^{5}$ These authors jointly supervised this work: André Cohnen, Wayne M. Coco.

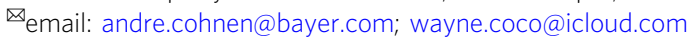


C RISPR systems evolved as a bacterial adaptive immune system in which resistance to phage infection is mediated by a nuclease (e.g., Cas9) that cleaves phage DNA. By exploiting the base-pairing potential of a guide RNA (gRNA), Cas9 targets a corresponding genomic locus for cleavage. A short motif on the targeted DNA, termed the protospacer adjacent motif (PAM), is necessary for Cas9 activity $^{1-4}$. CRISPR-Cas9 has been harnessed as a versatile tool for directed genome editing ${ }^{5-7}$ and, with the above constraints, allows the targeting in principle of any given chromosomal region of interest.

Streptococcus pyogenes Cas9 (SpyCas9), the most common enzyme used in genome-editing applications, is a large nuclease of 1368 amino acid residues ${ }^{5}$. The advantages of SpyCas 9 include its short, $5^{\prime}$-NGG-3' ${ }^{\prime}$ PAM and very high average editing efficiency. Despite concerns about its specificity profile in some applications, the listed features have led SpyCas9 to be explored among the CRISPR nucleases of choice for clinical gene therapy applications ${ }^{8}$.

Effective delivery of CRISPR-Cas systems to targeted cells and tissues is crucial for successful in vivo genome editing. For this purpose, recombinant adeno-associated virus (rAAV) vectors and lipid-nanoparticles (LNPs) are among the most prevalent and promising technologies ${ }^{9}$. Because packaging into rAAV vectors is limited to $\sim 5 \mathrm{~kb}$, Cas 9 proteins smaller than SpyCas9 are desirable to enable packaging of DNA encoding both Cas9 and sgRNA into one rAAV ("all-in-one-AAV") particle. This limitation is exacerbated for the larger multidomain-Cas-nuclease-based systems for base editing, prime editing, or CRISPRi/a ${ }^{10-14}$. Beyond rAAVs, smaller nucleases can also facilitate formulation and mRNA manufacturing for LNPs ${ }^{15}$. Smaller Cas9 proteins are thus of keen interest in the field.

The best-characterized smaller Cas9 are from Staphylococcus aureus (SauCas9, 1053 amino acid residues) ${ }^{16}$ and Campylobacter jejuni (CjCas9, 984 amino residues) ${ }^{17}$. However, both recognize longer PAMs, $5^{\prime}$-NNGRRT-3' for SauCas9 $(\mathrm{R}=\mathrm{A}$ or $\mathrm{G})$ and $5^{\prime}$ NNNNRYAC- $3^{\prime}$ for CjCas9 $(\mathrm{Y}=\mathrm{C}$ or $\mathrm{T})$, which reduces the number of uniquely addressable target sites in the genome, in comparison to the NGG SpyCas9 PAM. First reports indicate that SauCas9 specificity is similar to SpyCas $9^{18,19}$.

Protein engineering and directed protein evolution have been successfully applied to improve a wide range of properties of many therapeutic and nontherapeutic proteins, including SpyCas $9^{20-26}$. Gene family DNA shuffling is a powerful protein optimization approach that leverages sequence diversity from homologous genes by randomly swapping gene fragments or polymorphisms to generate screenable gene-variant libraries ${ }^{20,22,23}$. This allows a myriad of perturbations of protein structure and function, while simultaneously maintaining a relatively high fraction of functional clones in the libraries ${ }^{20-23}$. The compounded effects of the perturbations in each clone can have minor to major effects on the encoded protein's phenotype. Screening such a library can thus generate improvements involving combinations of a large number of simultaneous mutations, which are poorly accessible by other engineering approaches $^{21,22}$.

Here, we apply gene family shuffling to four small Cas9 nucleases ( $\sim 1050$ amino acid residues). Our aim was to generate short, but highly active and specific, synthetic RNA-guided nucleases (sRGNs, pronounced "surgeons") that recognize the favorable "GG" di-nucleotide PAM. Our resulting sRGNs displayed higher specificity and activity than SpyCas9 in human cell lines and robust in vivo editing in mice and nonhuman primates (NHP) when formulated as mRNA into LNPs, as well as when packaged as DNA into a single rAAV vector, thus making them well-suited candidates for gene therapy applications.

\section{Results}

Characterization of four related small Cas9 nucleases. We examined four related, previously uncharacterized Cas9s from Staphylococcus hyicus (Shy), Staphylococcus lugdunensis (Slu), Staphylococcus microti (Smi), and Staphylococcus pasteuri (Spa), and found three recognized a favorable "NNGG" PAM (Supplementary Fig. 1). Among these, only SluCas9 displayed prominent genome-editing activity using ribonucleoprotein particles (RNP) directed to the $H B B$ or the VEGFA locus in mammalian cell lines (Fig. 1a). The tracrRNA for all four parents were similar; however, upon assessing each of the four nucleases for activity with the SluCas9 tracrRNA, we observed increased genome editing only for ShyCas9 (Supplementary Fig. 2). The optimal guide length for SluCas9 was 21-23 nucleotides (Fig. 1b), similar to previous reports on SauCas9 ${ }^{16}$. Homology mapping to SpyCas9 and SauCas9 yielded predicted protein domains of the nucleases (Fig. 1c), facilitating the generation of nickases and inactive ("dead") enzymes (Supplementary Fig. 1e).

Protein engineering and characterization of improved nucleases. To improve the editing efficiency and specificity of SluCas9, we applied DNA family shuffling. We fragmented and reassembled the above four "parental" genes at multiple areas of high sequence identity, resulting in two combinatorial sRGN DNA libraries with a diversity of $8 \times 10^{3}$ and $1.3 \times 10^{5}$, respectively (Fig. 2a). To retain favorable $5^{\prime}-\mathrm{NNGG}-3^{\prime}$ PAM recognition, the PAM-interacting domain (PID) in all library variants was initially held constant as the native SluCas9-PID. These libraries were prescreened using a "live/dead" bacterial survival assay, yielding an enriched library of 1824 active nucleases, which were further narrowed to 165 hits with superior cleavage kinetics using a cell-free cleavage assay (Fig. 2b, Supplementary Fig. 3a, b). These resulting 165 hits were subsequently assayed for activity in mammalian cells by BFP gene disruption (Fig. 2c). In line with previous observations ${ }^{25}$, we found generally weak correlation between the activities of a given engineered variant in the different screening assays (Fig. 2d). We thus selected the two top hits for each assay, respectively designated sRGN1-4, for further engineering (Supplementary Fig. 4a).

Further improvements in performance were explored by incorporating alternative PIDs. As stated above, we initially replaced PID domains of wild-type ShyCas9 with alternative Slu-PID fragments, resulting in chimeric nucleases that acquired the ability to cleave a $5^{\prime}$-NNGG-3' PAM-containing target, in contrast to the Shy-native $5^{\prime}$-NNARMM-3' PAM (Supplementary Fig. 4b). This motivated replacement of respective C-terminal fragments of the selected sRGNs with analogous fragments from either SpaCas9 or SmiCas9, since both also recognize a $5^{\prime}$-NNGG-3' PAM. While tested SmiCas9 PID fusions were inactive, the SpaCas9 C-terminal fragments resulted in variable activity, depending on the recipient sRGN. All three SpaCas9-PID fragments resulted in active sRGN3 fusions, yielding sRGNs 3.1-3.3, and increased the median BFPcleavage activity up to 2.8-fold (Fig. 2e). Substituting these PID fragments in the other three top variants yielded no similar benefit (Fig. 2e).

To assess the overall performance with a diverse set of sgRNAs, we then selected sRGN1, sRGN2, sRGN4, and sRGN3.3 for further testing on 11 targets distributed within the BFP locus. BFP protospacers were selected for either "NNGG" PAM for sRGNs and "NGG" PAM for SpyCas9, resulting in target sites shifted by one nucleotide. While plasmid transformation comparisons are suboptimal for detailed quantitative comparisons, sRGN1, 2, and 4 showed significant activity on most targets. Variant sRGN3.3 demonstrated higher cleavage activity in this assay, comparable to the highly active SpyCas9 (Fig. 2f). 


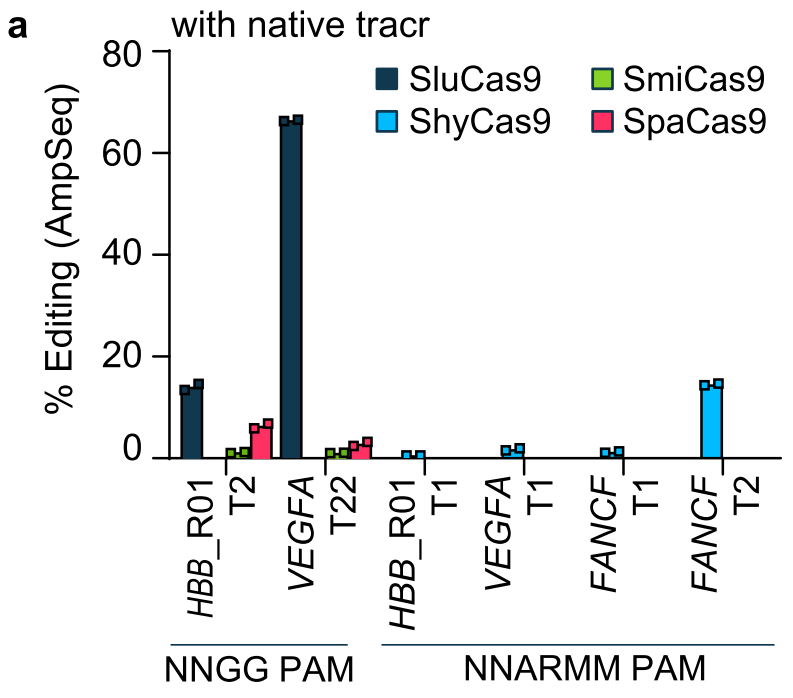

b

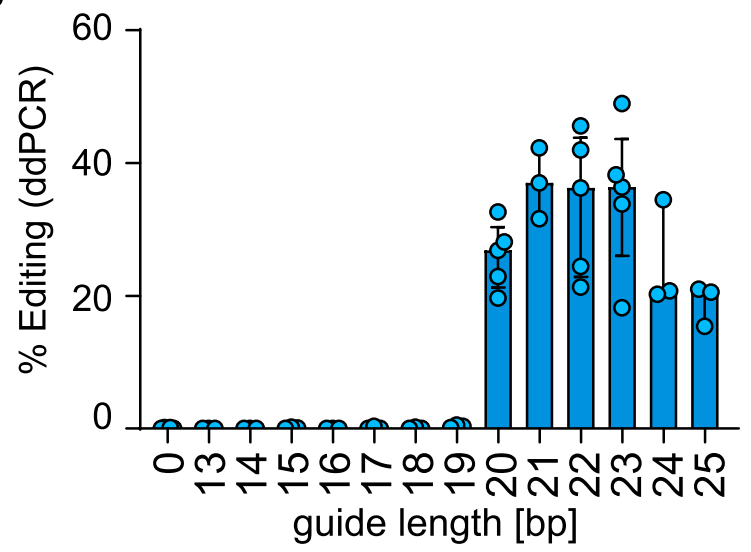

C
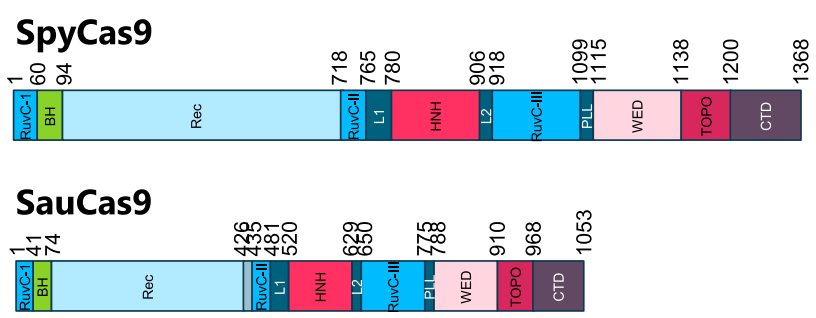

SluCas9

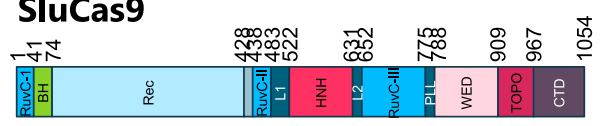

SpyCas9 has been characterized to be, for practical purposes, a single-turnover enzyme, while the smaller SauCas9 exhibits multiturnover activity ${ }^{26}$. In an in vitro assay with a twofold molar excess of substrate: RNP, sRGN3.1, and its parent, SluCas9, effected near-complete substrate-to-product conversion, indicating multiturnover capability. For SpyCas9, in contrast, we confirmed single turnover activity (Supplementary Fig. 5a).

With few exceptions, insertion/deletion (indel) pattern analysis of SluCas9, sRGN3.3, and SpyCas9 revealed similarities among these nucleases on the same targets (Supplementary Fig. 5b), supporting previous observations that indel formation pattern is more dependent on target locus than employed nuclease ${ }^{24}$.
Fig. 1 Genome editing and domain architecture of four Staphylococcus Cas9s. a Activity of wild-type Cas9s on HEK293T loci assayed by amplicon sequencing. Cas9s were tested as RNPs with their respective native tracr sequences and delivered via nucleofection. Spa, Smi, and Slu were tested on two targets with 5'-NNGG-3' PAM (guide_87 and guide_102 targeting the HBB_R01_T2 and VEGFA_T22 loci, respectively). Because of the distinct PAM for ShyCas9, the corresponding experiments with ShyCas9 required a different set of targets in these loci containing a 5'-NNARMM-3' PAM (guide_1-4 targeting the HBB_R01_T1, VEGFA_T1 and FANCF_T1, and FANCF_T2 loci). Data are presented as the mean of $n=2$ independent biological replicates. Editing values were background subtracted. $\mathbf{b}$ Effect of guide length on the efficiency of SluCas9 editing. To assess the optimal protospacer length for SluCas9, a single HBB site (R01_T2) was targeted by synthetic sgRNAs with protospacer lengths varying from 13 to $25 \mathrm{nt}$ (guide_267, 268, and guide_80-90) using RNP nucleofection. The editing efficiency was measured using ddPCR. For negative controls, the nuclease was nucleofected in the absence of sgRNA. Shown are individual measurements with the median as bars plus interquartile range, $n=3$ for length 13-19, 21, 24, and 25, $n=5$ for length 20, 22, and 23 independent biological replicates. c Domain architecture schematic for SpyCas962 and SauCas 963, and putative domain architecture for SluCas9 based on an alignment with SauCas9. Source data of $1 \mathrm{a}$ and $1 \mathrm{~b}$ are provided in the source data file.

Synthetic RNA-guided nuclease (sRGN) activity and specificity in cell-free and mammalian cell line assays. RNPs are attractive, therapeutically relevant entities for ex vivo cellular genome editing, especially due to their limited duration of activity resulting from the faster cellular turnover of Cas9 proteins vs. rAAV genomic DNA or plasmids. Using RNPs, we determined 22 nt as the optimal guide length for sRGN3.1 (Fig. 3a) and detected approximately two-fold higher potency for sRGN3.1 over SluCas9 at the HBB_R01 target in mammalian cells in gRNA titration experiments (Fig. 3b). Additionally, higher cleavage activity of sRGN3.1 and sRGN3.3 compared to SpyCas9 was observed on the albumin locus in a murine hepatoma cell line (Supplementary Fig. 6). We next assessed editing on a panel of endogenous, superimposed SpyCas9 and sRGN targets (adjacent to $5^{\prime}$-NNGGG-3' PAMs) on 24 diverse targets in HEK293T cells (Fig. 3c). The average editing efficiency across these targets was 2.3-fold (SluCas9) and 3.1-fold (sRGN3.1) higher than for SpyCas9 (Fig. 3c, right panel). Additionally, we performed a cellfree FP assessment on 48 synthetic target sequences engineered to have between 20 and $80 \%$ GC content, and on 48 additional therapeutically relevant human genomic targets. Overall, we found the protein-engineered sRGN3.1 outperformed SpyCas9 on 76 of these 96 targets and outperformed its most active parental nuclease, SluCas9, on 62 of 96 targets (Supplementary Fig. 7a).

Off-target (OT) cleavage is an important parameter in clinical applications. To initially characterize our sRGNs' propensities for OT cleavage, we analyzed their cleavage behavior in vitro using each possible single-nucleotide gRNA mismatch along the entire HBB_R01 target sequence (Supplementary Fig. 7b). Confirming previous reports ${ }^{27}$, we observed that SpyCas 9 showed low mismatch discrimination (low specificity) outside of its PAM-proximal "seed" region. SluCas9 showed overall specificity similar to SpyCas9 but with a less pronounced seed region and higher specificity in the PAM distal region. sRGN3.1 also lacked a prominent seed region, but instead showed an improved average level of mismatch discrimination across the entire protospacer region in comparison to SpyCas9.

To probe the specificity of sRGN3.1, SluCas9, and SpyCas9 in a human cell line, GUIDE-Seq ${ }^{28}$ was employed. We selected wellcharacterized guides targeting the VEGFA_T2 ${ }^{29}$ or HBB_R01_T2 $2^{30}$ locus and dosed RNP activity to achieve similar on-target editing 
a

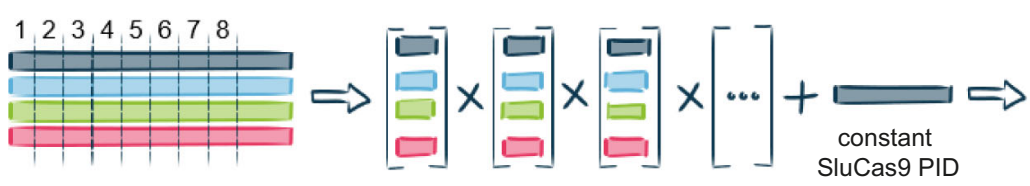

b

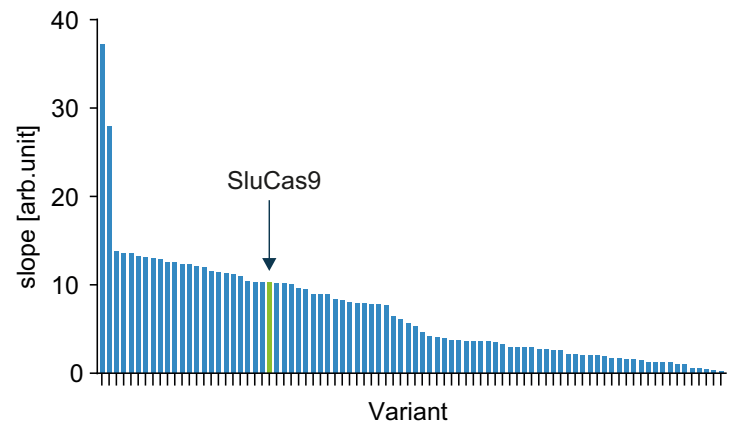

C

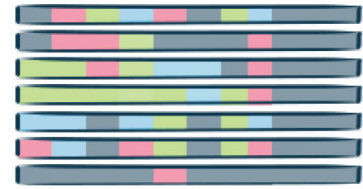

$[\ldots]$

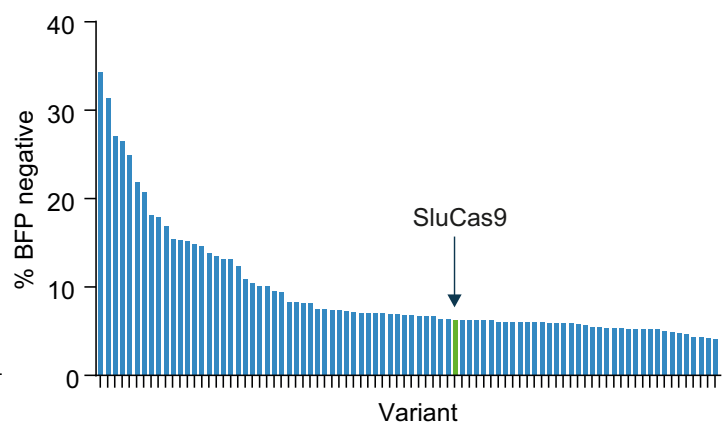

d

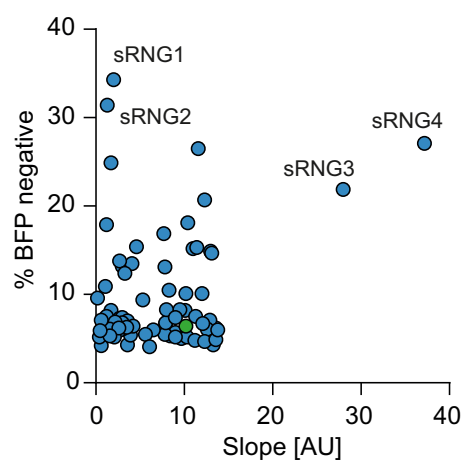

e

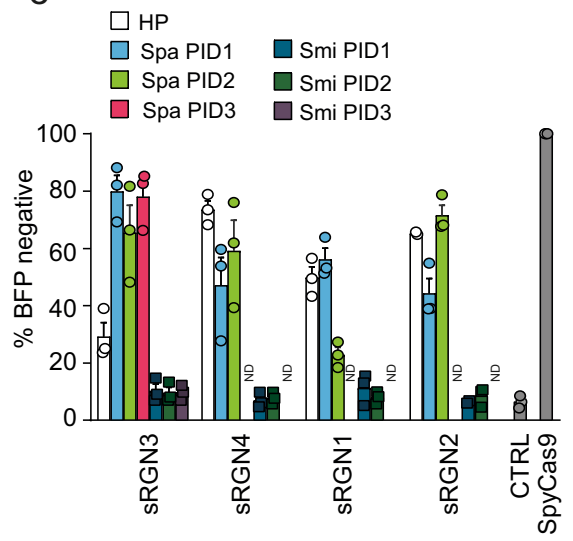

f

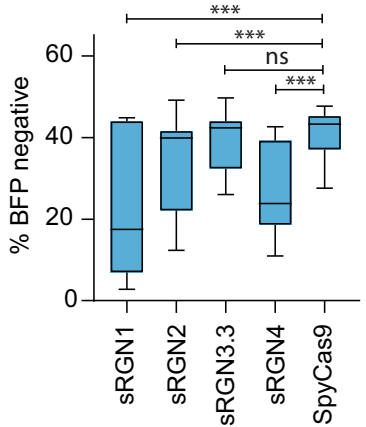

Fig. 2 Gene family DNA shuffling, screening, and confirmation of engineered, chimeric hits. a Schematic of the employed gene family DNA shuffling approach. Parental Cas9 gene fragments were generated with termini at 8 or 11 positions of high sequence identity and reassembled to yield gene family DNA-shuffled libraries containing synthetic RNA-guided nuclease (sRGN) genes with 8 or 11 fixed cross-over points. Each shuffled library member possessed a constant SluCas9 PI-domain (PID) to maintain NNGG PAM recognition. b Activity preselection using a "live/dead" bacterial survival assay, followed by a cell free-cleavage fluorescence polarization (FP) assay on the VEGFA_T2 target yielded 165 active chimeric nucleases. Slope values correlate with enzyme activity. A representative experiment is shown $(n=1)$, arb.unit $=$ arbitrary units. Green bar $=$ SluCas9 control. Source data are presented in the source data file. c HEK293T cells containing a genome-integrated BFP gene were transfected with plasmids encoding each of the 165 sRGN variants. The editing efficiency was assessed by the loss of BFP fluorescence. The gating strategy is shown in Supplementary Fig. 10. Data are presented as the mean of $n=2$ independent biological replicates. Green bar $=$ SluCas9 control. $\mathbf{d}$ Correlation of FP and BFP disruption data shown in $\mathbf{b}$ and $\mathbf{c}$ identified two top hits for each assay; sRGN1-4 were selected for further analyses, green dot = SluCas9. e SpaPID1-3 and SmiPID1-3 denote replacement of three alternative stretches comprising the constant Slu PID in the shuffled sRGN screening hits with corresponding parts of either SpaCas9 or SmiCas9. HP (hit PID) denotes the respective hit nuclease with its original PID. The values are normalized to SpyCas9. Data represent $n=3$ independent biological replicates with mean \pm SEM. $N D=$ not determined, CTRL = no enzyme. f BFP disruption data using 11 distinct targets (sRGNs guide_5-9 and 11-16, Spy guide_19-23 and 2530). Data are presented as minimum to maximum values; the box encompasses the 25th to the 75th percentiles, the line in the box is the median. $N=6$ independent biological replicates for each guide. Significance was determined by two-way ANOVA and Dunnett's multiple comparison test, ${ }^{\star \star \star} p<0.0001$, ns $=$ not significant $(p=0.8209)$. Source data are presented in the source data file.

for each nuclease (Supplementary Fig. 7d). As expected, the ontarget in each case yielded the highest number of reads. For SpyCas9, we recovered 66 unique off-targets using the VEGFA_T2 guide (of which 50 had been previously reported ${ }^{31}$ ) and 82 OTs using the $H B B \_$R01 guide. Consistent with our described cell-free observations, we found greatly reduced OT editing for SluCas9 (30 using VEGFA_T2, 2 for HBB_-R01) and an even greater specificity increase for sRGN3.1 (2 and 1 OTs, respectively). This was even the case in samples for which the extent of on-target editing was greater for sRGN3.1 and SluCas9 than for Spy. Extending the guide length from 20 to $22 \mathrm{nt}$ further reduced the number of OTs from 30 to 15 for SluCas9 on VEGFA_T2, and from 1 to 0 for sRGN3.1 on HBB_R01_T2 (Supplementary Fig. 7d and Fig. 3d). sRGN3.1 thus displayed significantly higher specificity than SpyCas9 in this context.

In vivo genome editing by sRGNs using LNP and AAV. Lipid nanoparticles are a current modality of choice to transiently deliver nucleases for genome editing applications in vivo. We thus evaluated the performance of sRGNs when delivered as mRNA in LNPs. Analyses using UPLC (Supplementary Fig. 8a) and 
a

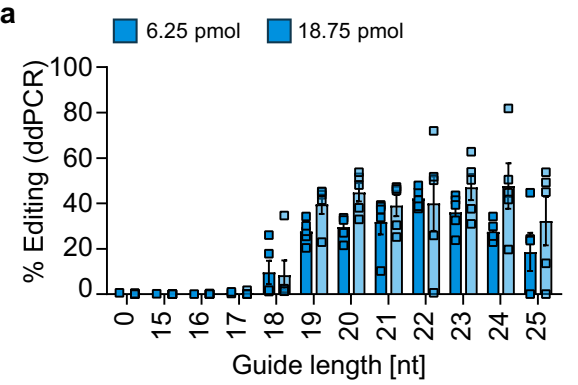

b

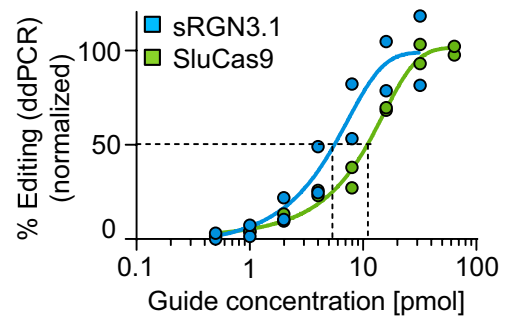

C

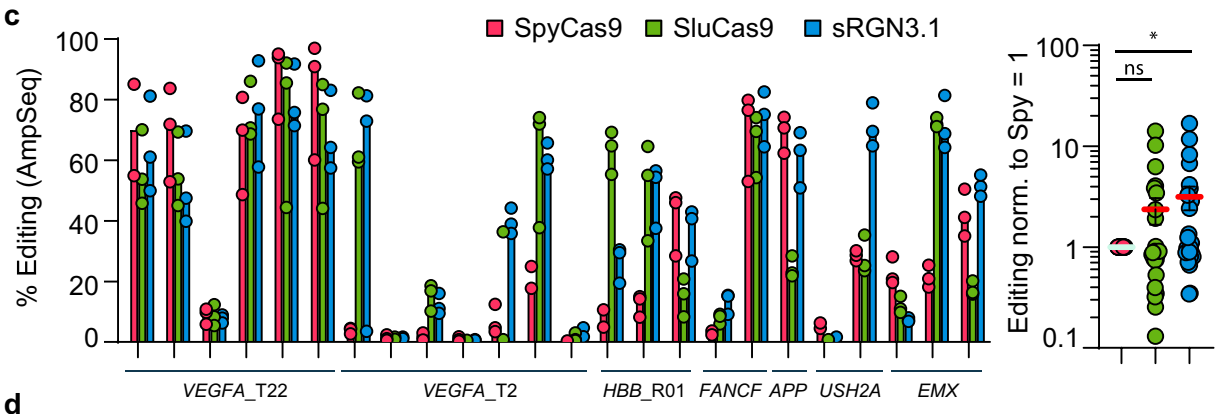

d

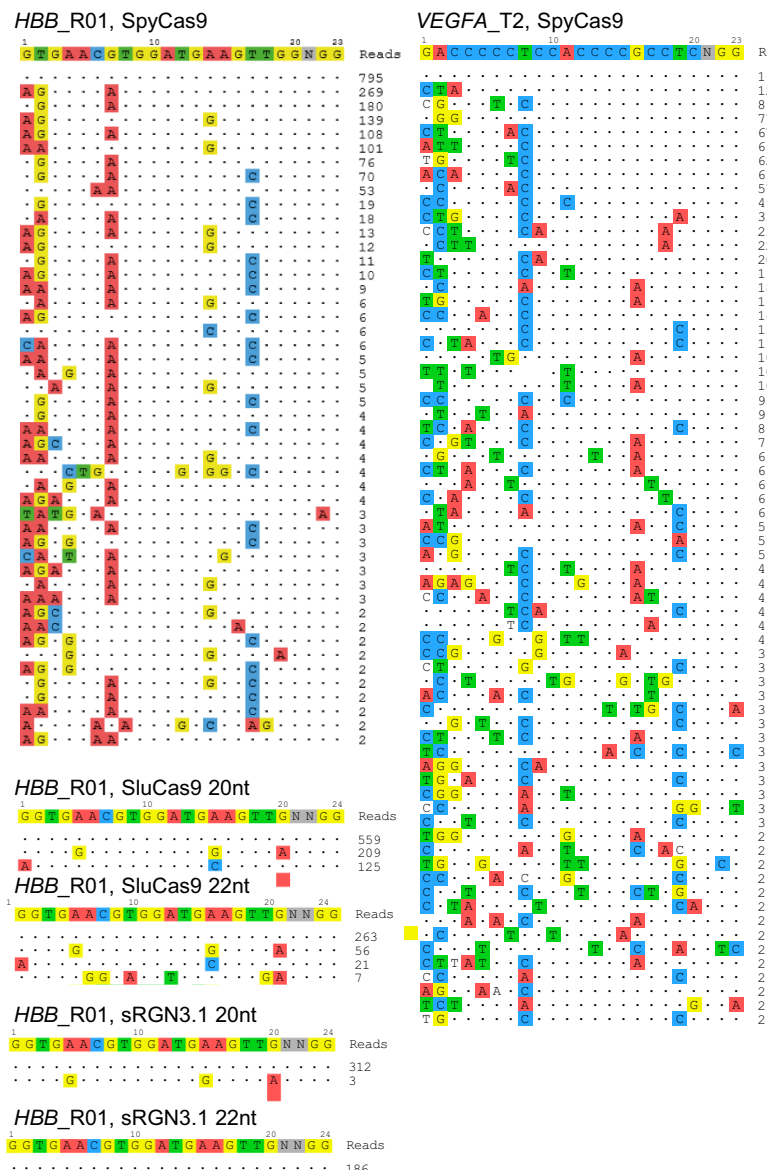

VEGFA_T2, SluCas9 $20 \mathrm{nt}$

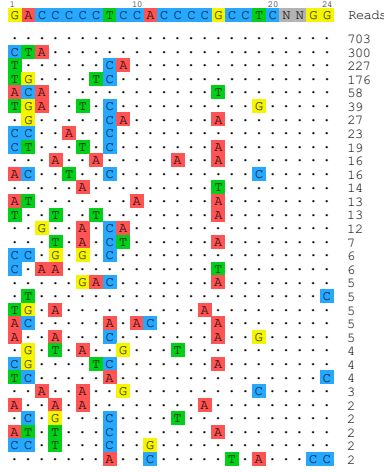

VEGFA_T2, SluCas9 $22 \mathrm{nt}$

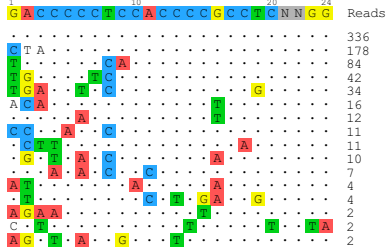

VEGFA T2, sRGN3.1 $20 \mathrm{nt}$

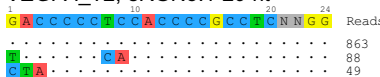

VEGFA_T2, sRGN3.122 nt

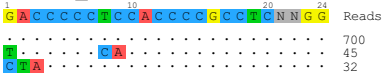

G GITGAACIG TG GIATGIA A G TT GN NG G Reads

cryoTEM (Supplementary Fig. 8b) showed differences in final lipid composition and improved LNP morphology, which appeared to be payload-dependent and which we hypothesize were due to the smaller sRGN mRNA. Interestingly, the sRGNLNPs also displayed enhanced functional stability, retaining potency significantly longer than SpyCas9-LNPs. Over 9 days of storage at $2-8^{\circ} \mathrm{C}$, a 3.6 -fold drop in editing efficiency was observed with SpyCas9-LNPs, whereas a potency drop of only 1.3-fold was observed with sRGN-LNPs, resulting in an over 3.6fold superiority in in vivo editing efficiency for the sRGN over SpyCas9 after 9 days of storage (Supplementary Fig. 8c).

We next compared LNP-mediated editing of the albumin locus by sRGNs and SpyCas9 in cells, as well as in the liver of C57BL/6 mice (Fig. 4a). Similar to the performance in cells (Supplementary 
Fig. 3 Genome editing efficiency and specificity of SluCas9 and sRGNs at endogenous loci in mammalian cells. a The effect of guide length on sRGN3.1 editing efficiency. The HBB_R01_T2 site was targeted by sgRNAs of lengths from 15 to 25 nts (guide_80-90) using RNP nucleofection and two different RNP concentrations. The editing efficiency was quantified by ddPCR; data are presented as mean \pm SEM with $n=3$ independent biological replicates. $\mathbf{b}$ Guide concentration dose-response. SluCas9 and sRGN3.1 activity after RNP nucleofection at the HBB_R01 locus (guide_87) in HEK293T cells. Curve fitting was conducted by the least-squares methods, $r 2=0.92$ (sRGN3.1) and 0.98 (SluCas9), the editing efficiency was measured by ddPCR and normalized to the maximum for each nuclease, data are presented as $n=2$ independent biological replicates. EC50s were 5.7 pmol for sRGN3.1 and 12.1 pmol for Slu. c The median activity on 24 genome targets. Comparison of genome editing efficiency at endogenous targets in HEK293T cells following RNP nucleofection. Twenty-four different guides (5'-NGGG-3' PAM), across seven genomic loci (VEGFA, HBB, FANCF, Apolipoprotein (APP), USH2A, and EMX), were assessed by amplicon sequencing (22 nt guides for SluCas9 and sRGN3.1: guides_32-55; 20 nt guides for Spy: guides_56-79, Supplementary Table 1). SpyCas9, SluCas9, and sRGN3.1 without guide RNA served as controls and had a median editing of $1.08 \pm 0.64 \%$; data are presented as $n=3$ independent biological replicates. The right data from the left panel with average editing on each target normalized to SpyCas 9 . Red line $=$ mean. The significance was determined using the one-sample Wilcoxon signed-rank test against a theoretical median of one with an alpha of $0.05, \mathrm{~ns}=$ not significant, $\left({ }^{\star}\right)=p \leq 0.05$, SpyCa9s vs SluCas9 $p=0.68$, SpyCas9 vs sRGN3.1 $p=0.05$. d GUIDE-Seq analysis for SpyCas9, SluCas9, and sRGN3.1 on HBB_R01 and VEGFA_T2. On-target sequences in $5^{\prime}-3^{\prime}$ orientation are shown in the top row of each panel. Identified off-targets are listed in the subsequent rows and ranked by the number of reads. Matches to the on-target are shown as (.), mismatches are highlighted. Note that for the SpyCas9 HBB_R01 off-target analysis, only 47 of the 82 detected off-targets are shown on distinct rows, because 37 of the determined off-target loci are distributed at two identical off-target sequences (AGgaacAtggatgaagCtgg was found 27 times, AGgaacAtggatgGagttgg 10 times). These duplicates were combined into the respective two rows. The full off-target list for each sample can be found in the source data file. Data are presented as $n=1$ for each guide.

Fig. 6), LNPs harboring sRGN3.1 mRNA displayed editing comparable to SpyCas9 in vivo, while sRGN3.3 was less efficient in this experiment. In addition, we tested the in vivo effects of mRNA sequence and chemistry modifications, including uridine depletion $^{32}$ and base substitution of uridine. Modification with (N1)-methylpseudouridine $(\mathrm{m} 1 \Psi)$ or with pseudouridine $(\Psi)$ had only modest influence on in vivo editing outcome (Supplementary Fig. 8d). Alternative modifications of the sgRNAs enhanced in vivo performance, with the utilization of a 23 -mer protospacer showing the largest effect on gene editing efficiency (Supplementary Fig. 8e).

In addition to LNP delivery, we evaluated AAV-based delivery, since the smaller size of sRGNs provides a packaging advantage over the larger SpyCas9. Usher syndrome type II (USH2) is an autosomal recessive disorder leading to hearing loss and retinitis pigmentosa ${ }^{33}$. This is caused by mutations in the USH2A gene, which is expressed in cochlear hair and retinal photoreceptor cells. One commonly found mutation in USH2 patients (termed IVS40) results in the insertion of an additional exon in USH2A mRNA, leading to premature truncation of the gene product ${ }^{33}$. We used sRGN3.1 and SpyCas9 to address the IVS40-specific mutation in an IVS40 homozygous cell line (referred to as 293FT-IVS40, also see Methods section). On-target editing in the 293FT-IVS40 line was higher for sRGN3.1 than for SpyCas9 (Supplementary Fig. 9b). Upon examining specificity by GUIDE-Seq and targeted amplicon sequencing of OT sites with up to seven nucleotide substitutions from the on-target site, we identified no OT editing above background for either SpyCas9 or sRGN3.1 (see amplicon sequencing data in the source data file, tab Supplementary Fig. 9a).

Toward clinical applications in humans, we tested the editing efficiency of rAAV-delivered sRGN3.1 in nonhuman primate (NHP) photoreceptors (PR) in vivo. As our NHP model possessed only the WT-USH2A allele, we pursued a surrogate strategy, assessing sRGN3.1 activity using a WT-specific USH2A guide (Supplementary Fig. 9b).

Of note, the larger size of SpyCas9 precludes an all-in-one approach. Comparison with SpyCas9 would require generation and co-injection of two rAAV5-genomes, one encoding the SpyCas9 nuclease and the second encoding the guide RNA expression components. Ethical considerations of a poorly comparable control in the NHP model thus led us to exclude SpyCas9 from this experiment.

The smaller size of sRGN3.1 allowed its packaging together with sgRNA into single AAV5 particles. We injected these particles into the NHP subretinal space and quantified indels in PRs. Six and twelve weeks after injection, we counted robust sRGN3.1-mediated in vivo editing in NHP-PRs (Fig. 4b). In summary, this result establishes successful AAV delivery of the compact sRGN3.1 gene, which was co-packaged with its sgRNA in single AAV vector particles, and allows for substantial genome editing in NHP photoreceptor cells.

\section{Discussion}

Here we report the generation and characterization of programmable small RNA-guided nucleases, which are smaller than SpyCas9, but with hgiher activity and specificity. Mouse studies with LNPs and AAV-mediated gene therapy in NHP show the potential of these additions to the CRISPR toolbox.

The packaging size limitation of AAV vectors and potential benefits from formulating smaller mRNA payloads into LNPs has generated considerable interest in genome-editing nucleases smaller than the widely used SpyCas $9^{34,35}$. However, these smaller alternatives to SpyCas9 typically show lower editing efficiencies on average across diverse targets in human cells and/ or possess more complex PAM sequences, which further limit their utility ${ }^{18,36,37}$. SauCas9 is similar in size to the presently described sRGNs, but has a less advantageous PAM, and engineered forms of SauCas9 struggle with lower activity on certain targets ${ }^{38}$. Of note, Kleinstiver et al. observed an average editing of $24.7 \%$ across 55 targets sites, while sRGN3.1 presented here shows an average editing of $41 \%$ across the 24 tested target sites. CjeCas9, although 74 amino acids smaller ${ }^{17}$, has not reached widespread use, potentially due to its long and degenerate PAM, which reduces the number of genomic sites targetable. Additionally, a recent paper in Science Advances ${ }^{39}$ raised important concerns about the enzyme, as it was shown to exhibit guideindependent, nonspecific host-cell DNA damage. In contrast, Nme2Cas9 is a small "CC" PAM nuclease. Importantly, unlike the sRGNs described in this work, the authors of the Nme2 paper find Nme2 inferior to SpyCas9 on 24 out of 28 sites tested ${ }^{40}$. Other recently published hypercompact enzymes such as Cas 14 and $\mathrm{CasPhi}^{41,42}$ are of interest in the field, even if no genomeediting activity on native (i.e., endogenous) genes in mammalian cells has yet been reported and no head-to-head comparison with SpyCas9 has been conducted. In addition, time fluorescence disruption activity assays like the ones reported here suggest they may be inferior to SpyCas9. Furthermore, we are not aware of reports outlining the specificity of CasPhi in a mammalian 
a

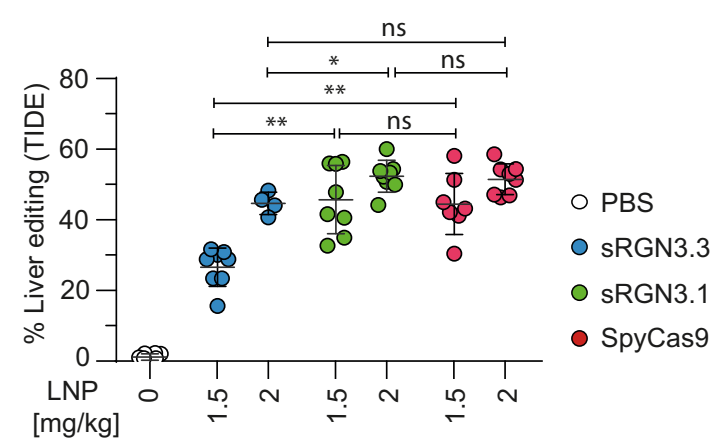

b
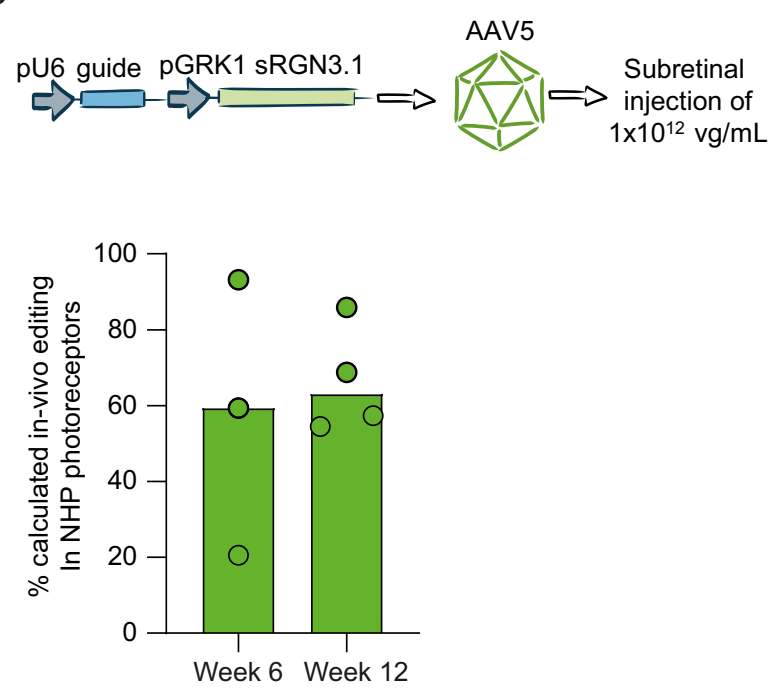

setting. Finally, the presence of a single nuclease domain in this protein makes it unlikely that it can be modified for the strandspecific nickase activity required for base editing or prime editing applications. By contrast, sRGN3.1, the product of this work, constitutes a chimeric dual-nuclease domain enzyme that recognizes a short $5^{\prime}$-NNGG- $3^{\prime}$ PAM, and which outperformed SpyCas9 regarding activity and specificity on the majority of tested targets.

We are aware of no reports to date of an alternative Cas9 nuclease with on-target editing efficiency comparable to SpyCas9 across a range of genomic targets. sRGN3.1 was determined to have a median activity $17 \%$ higher than SpyCas 9 on 24 targets selected for analysis only by virtue of an associated NGGG motif. Using LNPs delivered i.v. to the mouse liver, we demonstrated that the in vivo albumin-locus target activity of sRGNs was comparable to SpyCas9. However, this comparison employed a 20 nt guide for both nucleases, rather than the optimal guide length for sRGN3.1. In separate experiments (Fig. $1 \mathrm{~b}$ and Supplementary Fig. 8e) median sRGN activity in vivo was boosted significantly with $23 \mathrm{nt}$ guides. In addition, RNA base modifications were also shown to boost sRGN activity in vivo. Together, these results indicate sRGNs are an attractive payload that can be effectively delivered by LNPs for robust in vivo gene editing.

High specificity is of critical importance in gene therapy applications. The undesirable properties of off-target (OT) activity ${ }^{43,44}$ and the resulting undesired mutations and/or chromosome rearrangements ${ }^{45}$ have been attributed to the high activity of SpyCas9. Nevertheless, data on the small SauCas9 are suggestive that this lower-activity nuclease has similar specificity
Fig. 4 In vivo delivery of sRGNs by LNP or AAV to mouse and nonhuman primate. a Liver editing in mouse using sRGN-LNPs and SpyCas9-LNPs at doses of 1.5 and $2 \mathrm{mg} / \mathrm{kg}$. LNPs or PBS control was administered intravenously. Data are presented as mean $\pm \mathrm{SD}$, each dot represents an independent biological replicate; sRGN3.3: $n=8$ at $1.5 \mathrm{mg} / \mathrm{kg}$ and $n=4$ at $2 \mathrm{mg} / \mathrm{kg}$, sRGN3.1: $n=8$ at both doses, SpyCas9: $n=7$ at $1.5 \mathrm{mg} / \mathrm{kg}$ and $\mathrm{n}=8$ at $2 \mathrm{mg} / \mathrm{kg}$. All mRNA constructs were $\mathrm{m} 1 \Psi$ base-substitution modified. All mRNAs used in this work were of comparable quality: fulllength purity $\geq 85 \%$ and low dsRNA levels effected by reverse-phase purification. sRGN3.3 and sRGN3.1 used the same end-modified sgRNA against albumin (Alb-T1), while SpyCas9 used a similar sgRNA with a protospacer shifted by one nucleotide (to accommodate "NGG" PAM instead of "NNGG", sequences in Supplementary Table 1) and internal 2' Omethyl modifications (see Supplementary Fig. 8e). Significance was tested using the Kruskal-Wallis test (alpha $=0.05$ ) corrected for multiple comparisons using Dunn's test; ${ }^{\star} p<0.05 ;{ }^{\star \star} p<0.01$; ns = not significant (1.5 mg/kg: sRGN3.3 vs 3.1: $p=0.0027$, sRGN3.1 vs Spy: $p>0.9999$, sRGN3.3 vs Spy: $p=0.0053 ; 2 \mathrm{mg} / \mathrm{kg}:$ sRGN3.3 vs 3.1: $p=0.0449$, sRGN3.1 vs Spy: $p>0.9999$, sRGN3.3 vs Spy: $p=0.0780$ ). b top: schematic of the genetic construct, packaging, and delivery of AAV5 vector. Bottom: in vivo editing of nonhuman primate (NHP) photoreceptors by subretinal injection of rAAV5 vectors. AAV5 vectors carrying sRGN3.1 and WT-IVS40 sgRNA (guide_111) were injected into the subretinal space of NHPs. Indels were quantified by amplicon sequencing from retinal punches. Individual measurements and bar as mean, $n=3$ eyes at 6 weeks and $n=4$ eyes at 12 weeks, independent biological replicates. A previous study and our internal assessment demonstrated that nucleases driven by a photoreceptor-specific GRK1 promoter are expressed only by photoreceptors, which account for approximately $30 \%$ of the cells in retinal punches ${ }^{60,61}$. We therefore calculated the frequency of indels in the photoreceptor fraction with a multiplier of 3.3. Source data are provided in the source data file.

to SpyCas $9^{16}$. Our GUIDE-Seq experiments revealed specificity for sRGN3.1 that was markedly higher than for SpyCas9, despite both being titrated to similar on-target editing efficiency. Indeed, these observations were made in immortalized cell line backgrounds that have been shown to more sensitively reveal genomic OT propensities of editing nucleases than primary human cells $^{46-48}$. Interestingly, it appears that in engineering higher activity into sRGN3.1, we relaxed stringency at certain PAMproximal positions in comparison to SluCas9, the highest activity WT nuclease in our parental set, while simultaneously increasing PAM-distal specificity, at least for the target investigated in depth (Supplementary Fig. 7b). While sRGNs and SpyCas9 both performed well in discriminating the few genomic off-targets possessing one or two mismatches to the on-target gRNA (Supplementary Fig. 7b), there will typically be exponentially more genomic off-targets with three or four mismatches than with one or two. Despite the very high-specificity seed region of SpyCas9, the dispersed base-pairing requirement across the sRGN3.1 gRNA appeared to better serve it in discriminating offtargets with multiple mismatches (Fig. 7b, d). Although higher overall specificity was observed for sRGN3.1 in vitro, an additional hypothesis for its much higher specificity in mammalian cells may paradoxically include the engineered sRGN's higher activity: the requirement for fewer nuclease molecules per cell may contribute to the more rapid elimination of the nuclease and thus less opportunity for off-target effects.

Effective in vivo delivery of genome editing agents remains challenging for some applications, despite recent commercial successes ${ }^{34,49,50}$. We thus investigated LNP and rAAV in vivo delivery approaches for sRGN3.1. sRGN LNPs had comparable or improved analytical and stability profiles to SpyCas9-LNPs. 
Further investigation is required to understand if the improved properties observed can be attributed solely to the packaging advantages of smaller RNA payloads. The toxicity of LNP formulations has been shown highly dependent on administered lipid quantities ${ }^{51}$, which also may be improved with equal editing activity in smaller RNA payloads (higher RNA stoichiometry per unit LNP mass). In addition, RNA quality in such formulations is also key and mRNA cost and product quality are typically improved with shorter constructs ${ }^{52}$. The smaller sRGNs are thus also more attractive than SpyCas9 from a biopharmaceutical manufacturing and quality perspective.

AAVs present another clear case for the size-based advantages of sRGN payloads. The large size of SpyCas9 requires the packaging of Cas9 and its sgRNA into two separate AAV vectors, increasing manufacturing complexity and potentially decreasing therapeutic efficacy in some use cases ${ }^{53}$. We demonstrated that subretinal injection of sRGN3.1 as an all-in-one rAAV5 affected robust editing in NHP photoreceptors in vivo.

In summary, we engineered potent, small, genome-editing nucleases that recognize a favorable PAM. We demonstrated targeted genome cleavage efficiency equal or superior to the "activity gold-standard" SpyCas9, across a broad selection of targets in human cell lines. In addition, sRGNs showed markedly higher specificity in GUIDE-Seq experiments in human cell lines. Robust in vivo editing efficiency was observed for LNP delivery in mice and AAV5 delivery in NHP. For a given therapeutically relevant genomic locus, our data indicate that sRGN3.1 has a higher probability of being more active and more specific, while offering the delivery advantages of a smaller nuclease. We thus expect that these synthetic RNA-guided nucleases will be valuable additions to the current repertoire of CRISPR gene therapy nucleases, and look forward to the implementation of these enzymes in a range of applications.

\section{Methods}

Identification of Cas9 sequences. Four uncharacterized, putative Cas9 nucleases with $\sim 80 \%$ amino acid similarity and $\sim 65 \%$ amino acid identity from shotgun sequencing data in Uniprot for four Staphylococcus species were identified: $S$. hyicus (Shy) GB: CP008747.1, Uniprot: A0A418JLD8, S. lugdunensis (Slu) NCBI reference sequence: NZ_GL622352.1, Uniprot: A0A133QCR3, S. microti (Smi) GB: JXWY01000132.1, Uniprot: A0A0D6XNZ8, and S. pasteuri (Spa) GB: CP004014.1 (see also Supplementary Table 2). Direct repeat and tracr sequences were identified by inspection. We exploited GAAA tetraloop fusion of crRNA and tracrRNA, as previously reported ${ }^{5,16}$, to form single-guide RNAs (sgRNAs). DNA sequences for $\operatorname{sgRNAs}$ and nucleases were codon optimized and ordered at GeneArt and Twist Biosciences.

Screening of libraries. In an initial "live/dead" (L/D) cell survival selection in $E$. $\mathrm{coli}^{54}$, we depleted nonfunctional or low-activity variants (Supplementary Fig. 3b and 3c). E.coli BW25141( $\lambda$ DE3) cells harboring a plasmid-based arabinose-inducible ccdB reporter gene were co-transformed with an expression plasmid for sgRNA (targeting VEGFA-T2 within the ccdB reporter) and an IPTG-inducible nuclease expression plasmid. Cells were recovered for $60 \mathrm{~min}$ in SOB media and subsequently plated on LB selection plates (chloramphenicol and $10 \mathrm{mM}$ arabinose). Functional Cas9 variants cleaved the toxic $\mathrm{ccdB}$ reporter gene and led to cell survival under selection conditions. In all, 1824 functional clones from the $\mathrm{L} / \mathrm{D}$ selection were expressed in E. coli and lysates were individually tested for activity in a biochemical oligodeoxynucleotide (ODN) cleavage assay (Supplementary Fig. 3d).

\section{E. coli-based negative selection assay for identification of Cas9 PAM speci-} ficities. The negative selection for identification of PAM specificities of generated Cas9s was done as previously described ${ }^{54}$. Briefly, competent $E$. coli BL21Star $(\lambda D E 3)$ containing an IPTG inducible Cas $9\left(\mathrm{Amp}^{\mathrm{R}}\right)$ and a sgRNA $\left(\mathrm{Kan}^{\mathrm{R}}\right)$ expression plasmid were electroporated with a library of plasmids $\left(\mathrm{Cm}^{\mathrm{R}}\right)$ harboring a VEGFA_T2 target sequence with $3^{\prime}$ adjacent $\mathrm{N}_{7}$ PAM sequences. Plasmids containing functional PAMs are depleted when Cas9 is induced, whereas plasmids harboring nonfunctional PAMs remain uncleaved. Following a $60 \mathrm{~min}$ recovery in SOB media, transformations were plated on LB plates in two sets: one induced (ampicillin, kanamycin, chloramphenicol, and $0.2 \mathrm{mM} \mathrm{IPTG)} \mathrm{and} \mathrm{one} \mathrm{not} \mathrm{induced}$ (ampicillin, kanamycin, chloramphenicol) set. In order to exceed the complexity $(16,384)$ of the $\mathrm{N}_{7}$ library, approximately 100,000 CFUs were plated, and plasmids were isolated using Plasmid DNA Midiprep Kit (Qiagen). From the resulting plasmid libraries, a $271 \mathrm{bp}$ fragment covering the VEGFA_T2 target and $\mathrm{N}_{7}$ PAM was amplified by PCR using KOD Hot Start DNA Polymerase (Merck Millipore) with primers fw: 5'-CTCAGAAGTGAAACGCCGTAGCG-3' and rev: 5'CTTTTGAGTGAGCTGACACCGCTC-3' followed by a PCR-product purification step with QIAquick PCR Purification Kit (Qiagen). Single-indexed libraries were prepared using the TruSeq DNA PCR-Free High Throughput Library Prep Kit (Illumina). Next-generation sequencing (NGS) was performed using a MiSeq reagent kit v3 with 600 bp paired-end sequencing on an Illumina MiSeq Sequencer. The resulting raw FASTQ files from the MiSeq runs were analyzed with a python script to determine relative PAM depletion. Shortly, this involves scripts to (1) process crude NGS Data for VEGFA_T2 target sequence [Each FASTQ entry is scanned for 12 constant nucleotides on the library amplicon on both strands. If the constant region is found, then the seven variable nucleotides flanking the protospacer region are captured and multiples are counted.]; (2) calculate depletion values by comparing PAM sequence motifs of depleted samples to undepleted controls and; (3) rank sequences according to frequency.

Fluorescence polarization assay. Oligonucleotide duplexes (oligo sequences are listed in Supplementary Table 3) were prepared in $1 \times \mathrm{PBS}+5 \mathrm{mM} \mathrm{MgCl}_{2}$ as $10 \mu \mathrm{M}$ solutions (from $100 \mu \mathrm{M}$ stocks) by melting at $95^{\circ} \mathrm{C}$ for $5 \mathrm{~min}$ and then slowlycooling to room temperature (RT). The stocks were subsequently diluted in $1 \times \mathrm{PBS}+5 \mathrm{mM} \mathrm{MgCl}+2.05 \%$ pluronic F68 (Sigma Aldrich) to $20 \mathrm{nM}$ working solutions. Twenty microlitres $(20 \mathrm{nM})$ of dsDNA was immobilized on a streptavidin-coated plate (Greiner, 384-well) and incubated for $10 \mathrm{~min}$ at RT. The plate was washed twice with 1X PBS and was subsequently incubated with $20 \mu \mathrm{L}$ of a diluted lysate supplemented with $60 \mathrm{nM}$ sgRNA targeting the VEGFA_T2 target sequence. Cas9 variants were expressed in $800 \mu \mathrm{L} \mathrm{TB}$ (Terrific Broth)-medium, as described in the protein expression and purification section, at the 96-well expression scale. Cells were harvested and lysed in $120 \mu \mathrm{L}$ lysis buffer (1× PBS + $0.5 \times$ BugBuster $^{\oplus}+5 \mathrm{mM} \mathrm{MgCl}_{2}$ ) and incubated for $10 \mathrm{~min}$ at $\mathrm{RT}$ under shaking conditions. The lysate was cleared by centrifugation: $3166 \times g, 10 \mathrm{~min}$ at $4{ }^{\circ} \mathrm{C}$. Cleared lysate was diluted $1: 3.5(\mathrm{v} / \mathrm{v})$ in $1 \times \mathrm{PBS}+5 \mathrm{mM} \mathrm{MgCl}_{2}$ and mixed with $60 \mathrm{nM}$ sgRNA, prior to a $5 \mathrm{~min}$ incubation at $37^{\circ} \mathrm{C}$. Cleavage was monitored by following both decreasing anisotropy and increasing fluorescence intensity for $60 \mathrm{~min}$ (excitation wavelength: $635 \mathrm{~nm}$; emission wavelength: $670 \mathrm{~nm}$ ) at $37^{\circ} \mathrm{C}$ in a plate reader (Tecan Infinite M1000 pro). For activity specificity assessment of purified protein, prior to the kinetic measurement, RNPs were formed in $1 \times \mathrm{PBS}+$ $5 \mathrm{mM} \mathrm{MgCl}$, with a two-fold excess of sgRNA at $37^{\circ} \mathrm{C}$ for $5 \mathrm{~min}$ with adjusted protein levels for the same activity. To obtain an overall specificity value, the cleavage kinetics were analyzed by calculating the initial slope of the reaction. The slopes were calculated for each of the 61 substrates (including the un-modified sequence and all single-nucleotide exchanges along the VEGFA_T2 target sequence) and normalized to the value of the on-target substrate. For positionspecific nucleotide tolerance profiles, the cleavage kinetics were analyzed by calculating the initial slope of the oligonucleotide cleavage reaction. The slopes were calculated for each of the 61 substrates and normalized to the value of the on-target substrate (defined as 1). The normalized cleavage values for all 60 off-target substrates were grouped according to their position. For each of the 20 nucleotide positions in the target sequence, the normalized cleavage value of the three single nucleotide mismatches was plotted to illustrate the position-specific nucleotide tolerance of the Cas9 protein.

Expression and purification of Cas9 proteins. Cas9 proteins were expressed from a plasmid harboring a TRC-promoter expression cassette, encoding an $\mathrm{N}$-terminal 6xHis-MBP-TEV fusion followed by a nucleoplasmin NLS and a C-terminal SV40 NLS. E. coli BL21 (DE2) star was transformed with the expression construct and a single colony was grown overnight at $37^{\circ} \mathrm{C}$ in $\mathrm{ZY}$-medium (including $21.5 \mathrm{ml}$ of $52 \times 5052$ solution ( $0.5 \%$ glycerol, $0.05 \%$ glucose, and $0.2 \%$ a-lactose), $52.5 \mathrm{ml}$ of 20xNPS, and $2 \mathrm{mM} \mathrm{MgSO}_{4}$ ) supplemented with $100 \mu \mathrm{g} / \mathrm{mL}$ ampicillin. One liter of supplemented ZY-medium was inoculated 1:100 (v/v) with the overnight culture and shaken at $37^{\circ} \mathrm{C}$ for $3 \mathrm{~h}$. After $3 \mathrm{~h}$, the culture was cooled to $18^{\circ} \mathrm{C}$ and incubated overnight. IPTG in a final concentration of $1 \mathrm{mM}$ was added, and the culture was incubated for two additional days. The cells were harvested by centrifugation $\left(4376 \times g, 20 \mathrm{~min}, 4^{\circ} \mathrm{C}\right)$, the cell pellet was resuspended in $22 \mathrm{~mL}$ wash buffer $(50$ $\mathrm{mM} \mathrm{Na}_{2} \mathrm{HPO}_{4} 300 \mathrm{mM} \mathrm{NaCl}, 10 \mathrm{mM}$ imidazole, $\mathrm{pH}$ 8.0) and lysed under sonification $10 \mathrm{~min}$ on ice (amplitude 30). The lysate was cleared by centrifugation $\left(194300 \times g, 30 \mathrm{~min}, 4^{\circ} \mathrm{C}\right)$ and applied to His-Trap HP columns (GE healthcare) using the ÄKTA pure system (GE healthcare) to perform an immobilized metal affinity chromatography (IMAC) step. After two washing steps, first with wash buffer including $0.1 \%$ triton, then with wash buffer only, the protein was eluted with a linear gradient of $10-500 \mathrm{mM}$ imidazole. Pooled protein fractions were subsequently treated with TEV at $4{ }^{\circ} \mathrm{C}$ for 2 days to cleave off the His-MBP tag. After $48 \mathrm{~h}$, the protein solution was buffer exchanged into equilibration buffer (20 mM HEPES, $100 \mathrm{mM} \mathrm{KCl}, \mathrm{pH}$ 7.0) using PD-10 columns (GE healthcare) and a cation exchange chromatography was performed by using HiTrap SP HP 
columns (GE healthcare) with a linear gradient of $100-1000 \mathrm{mM} \mathrm{KCl}(20 \mathrm{mM}$ HEPES, $1000 \mathrm{mM} \mathrm{KCl}, \mathrm{pH}$ 7.0). Before protein fractions were pooled, quality and purity were checked via SDS-PAGE, and a biochemical cleavage assay was conducted to exclude fractions with inactive nuclease contaminations. Nuclease activity used in downstream assays was standardized by sgRNA titrations. Briefly, starting from an excess of sgRNA, the amount of sgRNA was reduced, until a decrease in activity was observed. Fractions were pooled, concentrated, and stored in storage buffer (20 mM HEPES, $200 \mathrm{mM} \mathrm{KCl,} 1 \mathrm{mM} \mathrm{DTT}, 40 \%$ glycerol pH 7.0) at $-20^{\circ} \mathrm{C}$.

Multiple-turnover analysis. RNP formation was performed with $20 \mu \mathrm{L}$ of gRNA ( $800 \mathrm{nM}$ stock) in $20 \mu \mathrm{L}$ of $1 \mathrm{x}$ NEB buffer and heated to $90^{\circ} \mathrm{C}$ for $3 \mathrm{~min}$ and cooled to $25^{\circ} \mathrm{C}$ at $1 \mathrm{C} / \mathrm{s}$. RNP was formed by combining $20 \mu \mathrm{L}$ of this gRNA with $20 \mu \mathrm{L}$ of Cas9 in 1x NEB buffer at a stock concentration of $200 \mathrm{nM}$ the final concentration was $100 \mathrm{nM}$ Cas and $200 \mathrm{nM}$ gRNA. The concentration of active Cas9 was determined ${ }^{55}$ before kinetic analysis for enzymes that were obtained commercially or synthesized in house. This solution was incubated at $25^{\circ} \mathrm{C}$ in a thermal cycler for $30 \mathrm{~min}$ prior to use. On-target reactions: $8 \mu \mathrm{L}$ of off-target plasmid $(250 \mathrm{nM}$ stock) was combined with $10 \mu \mathrm{L}$ of $10 \mathrm{x}$ NEB buffer and $72 \mu \mathrm{L}$ of RNase-free water. This solution was placed on a heat block at $37^{\circ} \mathrm{C}$ and the reaction was initiated by adding $10 \mu \mathrm{L}$ of the RNP stock. Quenching and analysis: At various time points, $5 \mu \mathrm{L}$ of the reaction was removed and quenched with $2 \mu \mathrm{L}$ of a $500 \mathrm{mM}$ EDTA solution. Once all samples were collected, RNP was removed by adding $1 \mu \mathrm{L}$ of proteinase K to each reaction. Samples were analyzed on an Agilent 2100 Bioanalyzer using a DNA 7500 kit.

Cell culture. HEK293T cells (ref. CRL-3216) and murine Hepa 1-6 cells (ref. CRL1830) were purchased from ATCC and 293FT cells were purchased from Thermo Fisher Scientific (ref. R70007).

HEK293T, 293FT, and homozygous 293FT-IVS40 cell lines were cultured in DMEM-GlutaMAX (Gibco) supplemented with 10\% FBS (Gibco), and 1\% sodium pyruvate (Gibco) and were cultured at $37^{\circ} \mathrm{C}$ in $5 \% \mathrm{CO}_{2}$. Hepa $1-6$ cells were cultured in DMEM (Gibco) supplemented with 10\% FBS (Gibco) and 1\% penicillin-streptomycin (Gibco) at $37^{\circ} \mathrm{C}$ with $5 \% \mathrm{CO}_{2}$

293FT-IVS40 cell lines were constructed as follows: to introduce the single nucleotide substitution corresponding to IVS40, TrueCut Cas9 Protein v2 complexed with TrueGuide synthetic sgRNA (Thermo Fisher Scientific) and 200nucleotide ssODN (5'-TCAGCCAGAGCAGGAAGCTAATAAAATGTATGCTG GCTTTTAAGGGGGAAACAAATCATGAAATTGAAATTGAACACCTCTCCT TTCCCAAGGTAAGAGATCATCTTTAAGAAAAGGCTGTGTATTGTGGGGG TTTGAAGTGCAAGTTCATCTCATTATCATGGATGTTTCACCCATAATACT ATCATCATATGCAGGAG - $3^{\prime}$ ) were used for stable transfections. Transfected cells were cultured as serial dilutions and screened by Sanger sequencing to isolate homozygous clones.

Plasmid, mRNA, and RNP transfection, harvesting, and lysis. For BFP disruption assay and indel pattern analysis, 15,000 HEK 293T cells/well were seeded the day before transfection in poly-D-lysine-coated 96-well plates (Biocoat Poly-D-Lysine, Fisher Scientific). On the next day, a medium change was performed, which was followed by the transfection of $140 \mathrm{ng}$ Cas9- or sRGNexpression plasmid plus $60 \mathrm{ng}$ gRNA-expression plasmid using LipoD293 (SignaGen) as a transfection reagent according to the manufacturer's instructions. The medium was changed $24 \mathrm{~h}$ later. Seventy-two hours post transfection medium was removed, and cells were trypsinized in $25 \mu \mathrm{L}$ TrypLE (Gibco) for $15 \mathrm{~min}$ at $37^{\circ} \mathrm{C}$ and diluted with $100 \mu \mathrm{L}$ PBS. Ten microliters of the sample was lysed by adding 0.5 $\mu \mathrm{l}$ DNA Release additive (B93, Thermo Fisher Scientific) and $20 \mu \mathrm{L}$ Dilution buffer (F1325ML, Thermo Fisher Scientific) and incubated for $5 \mathrm{~min}$ at room temperature followed by $98^{\circ} \mathrm{C}$ for $2 \mathrm{~min}$. For mRNA transfection, murine Hepa 1-6 cells were plated in 24-well plates at a density of 50,000 cells per well. After incubation for $2 \mathrm{hr}$, cells were transfected with $0.25 \mu \mathrm{g}$ of nuclease mRNA and $0.25 \mu \mathrm{g}$ of sgRNA per well using Lipofectamine MessengerMAX (Thermo Fisher Scientific) by following the manufacture' $r$ 's instructions. Cells were collected 3 days after transfection and genomic DNA was extracted using the DNeasy Blood \& Tissue Kit (Qiagen) following the manufacturer's instructions. Parental 293FT and the homozygous IVS40 293FT cell line $\left(2 \times 10^{5}\right.$ cells $)$ were transfected with $2 \mu \mathrm{g}$ of a sRGN3.1 expression plasmid and with either T428 sgRNA or T428 surrogate sgRNA by using Lipofectamine 3000 (Thermo Fisher Scientific Scientific). Genomic DNA was extracted 7 days after transfection with DNeasy Blood \& Tissue Kit (Qiagen). A fragment containing the editing region was amplified using PCR with the appropriate primers (Supplementary Table 3) and used for TIDE analysis.

For Ribonucleoprotein (RNP) complex formation, crRNA and tracrRNA (IDT, phosphorothioate bonds, and 2'-O-methyl groups at the last two nucleotides, see Supplementary Table 1 for sequences) were dissolved in IDTE buffer at $200 \mu \mathrm{M}$ and mixed at equimolar ratios to obtain $100 \mu \mathrm{M}$ working solution. The crRNA: tracrRNA duplex was incubated at $95^{\circ} \mathrm{C}$ for $5 \mathrm{~min}$ and gradually cooled to $4{ }^{\circ} \mathrm{C}$. To prepare Cas9/sRGN-RNP complexes, Cas9/sRGN protein was incubated with crRNA:tracrRNA duplex at a 1:2 molar ratio. Cas9/sRGN and RNA complex was incubated in $10 \mathrm{mM}$ TRIS at RT for $15 \mathrm{~min}$ to form RNP complexes. $2 \times 10^{5}$ cells were washed with 1X PBS (Gibco), detached with Accutase (Sigma), spun down by centrifugation at $300 \mathrm{xg}$ for $5 \mathrm{~min}$, washed again with 1X PBS and nucleofected with precomplexed RNPs using the 4D nucleofector with the SF cell line kit and program CA-137 (Lonza), according to the manufacturer's protocol. Cells were seeded on poly-D-lysine-coated 24-well plates (Biocoat Poly-D-Lysine, Corning) and harvested 2-3 days after nucleofection by 15 min TrypLE (Gibco) incubation at $37^{\circ} \mathrm{C}$. Genomic DNA from RNP-treated cells was extracted with QIAamp DNeasy Blood \& Tissue Kit (Qiagen) according to the manufacturer's instructions.

BFP disruption assay. This assay was adapted based on the previously described traffic light assay ${ }^{56}$. Briefly, HEK293T cells harboring a gene encoding BFP in the AAVS1 locus were transfected with plasmid expressing Cas9 and a sgRNA targeting the reverse strand of BFP genomic sequence (guide_5 for initial screens). Transfection of plasmids was performed using Lipofectamine 3000 (Thermo Fisher Scientific, Cat no. L3000015) as described by the supplier. Flow cytometric analyzes of BFP signal disruption was carried out 7 days posttransfection as follows. Cells were prepared by washing with $1 \mathrm{X}$ PBS, trypsinization, and resuspension in $200 \mu \mathrm{L}$ FACS buffer (1X PBS supplemented with 2\% FCS). BFP signal from minimum of 10,000 cells was assayed using the V450 filter set in the BD FACS Canto II (see Supplementary Fig. 1 for gating strategy). For BFP landscaping analysis sRGNs guide_5-17 and Spy guide_19-31 were used. We switched from the initial T7 promoter spacer $\left(5^{\prime}\right.$-TATA- $\left.3^{\prime}\right)$ to the more canonical U6 spacer $\left(5^{\prime}\right.$-AAACACC- $\left.3^{\prime}\right)$ for these experiments. Gating strategy is presented in Supplementary Fig. 10.

Amplicon sequencing (AmpSeq) for on- and off-target analysis and NGS analysis. To assess the editing efficiency or to evaluate predicted off-targets, a $\sim 200-240 \mathrm{bp}$ fragment flanking the genomic target site was amplified by PCR using Q5 HighFidelity polymerase (2XMM Master Mix (NEB), $1 \mu \mathrm{L}$ crude cell lysate or $50 \mathrm{ng}$ purified genomic DNA, and barcoded primers (see Supplementary Table 3 for locus-specific primers). The cycling program was performed according to the manufacturer's instructions with an annealing temperature of $66^{\circ} \mathrm{C}$ and an extension time of $15 \mathrm{~s}$. All PCR reactions were pooled into a library and purified with magnetic beads (AMPure XP beads, Beckman Coulter). Bead purification was performed by applying the following reaction steps. Two micrograms of the pooled amplicons were end-repaired and dA-tailed (NEBNext Ultra II End Repair/dAtailing Module, NEB) and used for ligation of Illumina indices (Blunt/TA Ligase master Mix, NEB). The concentration was measured using the dsDNA HS Kit (Thermo Fisher Scientific) on a Qubit spectrophotometer and diluted to $4 \mathrm{nM}$. The library was denatured with $0.2 \mathrm{M} \mathrm{NaOH}$ for $5 \mathrm{~min}$ at RT and diluted to $10 \mathrm{pM}$ using the buffer provided in the MiSeq 300PE v2 Kit. The sample was sequenced with 12.5 pmol PhiX on a MiSeq Instrument (Illumina). For de-multiplexing an inhouse python script was used and individual samples were analyzed using CRISPResso V1.0.13 ${ }^{57}$ to extract \% NHEJ. A threshold of $>20,000$ reads was chosen.

Evaluation of off-target sites at the USH2A locus. Forty-nine sites containing up to five nucleotide substitutions from the T428 target sites were selected for analysis. The homozygous IVS40 293FT cell line $\left(2 \times 10^{5}\right.$ cells $)$ was transfected with $2 \mu \mathrm{g}$ of a plasmid carrying sRGN3.1 and T428 sgRNA in Lonza SF buffer by nucleofection under Program CM-130, and genomic DNA was extracted 7 days after nucleofection. All the selected sites except one on-target site were successfully PCRamplified, and indels were analyzed by NGS as described in the section Amplicon sequencing (AmpSeq) and NGS analysis.

Digital droplet PCR. In order to determine editing rates, ddPCR (Bio-Rad) was performed with $50 \mathrm{ng}$ genomic DNA by using ddPCR supermix for probes (no dUTP, Biorad). Droplet formation was accomplished by (Biorad) QX200 Droplet Generator Amplification was done by using the following primers, probes, and annealing temperatures in a 40 cycle program with an additional extension step of $72^{\circ} \mathrm{C}$ (HBB_R01: amplification primer fw $5^{\prime}$-catggtgcatctgact cctg- $3^{\prime}$, rev $5^{\prime}$-ggtagaccaccagcägccta- $3^{\prime}$, NHEJ-sensitive-probe $5^{\prime}$-TGAAGTTG GTGGTGAGGCCCT-3', reference probe 5'-AGGAGAAGTCTGCCGTTACTG CCCT- $3^{\prime}$, annealing temperature $58.5^{\circ} \mathrm{C}$ ). Analysis was performed on the QX200 ddPCR Reader (Biorad).

GUIDE-seq analysis. GUIDE-seq experiments were performed as previously described $^{28}$. For off-target analyses on the USH2A (IVS40) locus, the homozygous IVS40 293FT cell line $\left(2 \times 10^{5}\right.$ cells) was nucleofected with 5 pmol of dsODN and $2 \mu \mathrm{g}$ of a plasmid carrying sRGN3.1 and T428 sgRNA in Lonza SF buffer by nucleofection under Program CM-130. Genomic DNA was extracted 3 days after nucleofection using the PureLink Genomic DNA Mini Kit (Thermo Fisher Scientific Scientific), and processed for sequencing as previously described ${ }^{28}$

Briefly, HEK293T cells were transfected with nuclease and sgRNA as RNPs as described above with addition of 5 pmol phosphorothioate (PS) end-protected double-stranded oligonucleotide (dsODN). For VEGFA_T2 and HBB_R01 offtarget analysis we observed higher activities of SluCas9 and sRGN3.1 compared to SpyCas 9 on these targets. Therefore, we used 60 pmol SpyCas 9 and 30 pmol each of SluCas9 and sRGN3.1 for targeting HBB_R01, except for sRGN3.1 with $22 \mathrm{nt}$ guide, 8 pmol were used. For targeting VEGFA_T2, 60 pmol SpyCas9, 8, and 18 pmol sRGN3.1 (for $20 \mathrm{nt}$ and $22 \mathrm{nt}$ guide, respectively) and 6 and 10 pmol SluCas9 (for $20 \mathrm{nt}$ and $22 \mathrm{nt}$ guide, respectively) were used to obtain a similar extent of 
on-target editing for each nuclease (Supplementary Fig. 7c). Genomic DNA was extracted $48 \mathrm{~h}$ upon nucleofection with DNeasy Blood \& Tissue Kit (Qiagen) and on-target activity of nucleases was evaluated via amplicon sequencing as described above. Genomic DNA was quantified by Qubit dsDNA BR assay (Invitrogen) and 400 ng sample was sheared with NEBNext UltraII FS enzyme mix (NEBNext UltraII FS DNA Library Prep Kit, NEB) for $10 \mathrm{~min}$ at $37^{\circ} \mathrm{C}$, followed by $30 \mathrm{~min}$ inactivation at $65^{\circ} \mathrm{C}$. Sample libraries were constructed with NEBNext Ultra II DNA Library Prep Kit (NEB) with adapters and primers previously described ${ }^{28}$. Sample libraries were sequenced with Illumina MiSeq. Similar read-depth samples were analyzed with the open-source software package guideseq ${ }^{28}$ (https://github. com/aryeelab/guideseq commit version c608522), using human genome assembly GRC37/hg19 as reference.

TIDE analysis. Evaluation of editing efficiencies on the USH2A and Alb locus was done via TIDE (Tracking of indels by decomposition) ${ }^{58}$ analysis. Targeted-specific amplicon products were generated with $50-100$ ng template DNA and primers spanning the target site (listed in Supplementary Table 3). Indels were identified via decomposition of quantitative trace data with the TIDE software (https://tide.nki.nl), using default parameters.

LNP formulation and characterization. The LNPs used in this study comprised a lipid mixture consisting of C12-200 (amino lipid, Axolabs), 1,2-dioleoyl-snglycero-3-phosphoethanolamine (DOPE), DMPE-mPEG2000 (PEG-lipid), and cholesterol at a 52.2:15.6:8.7:23.6 mass ratio, respectively (DOPE, PEG-lipid, and cholesterol from Avanti Polar Lipids). LNPs were prepared by rapid microfluidic mixing (Precision NanoSystems) of mRNA and sgRNA in acetate buffer at $\mathrm{pH} 4.0$ with the lipid mixture suspended in ethanol (3:1 aqueous to organic volume ratio). After mixing, LNPs were diluted and dialyzed into PBS, concentrated as needed using 100k MWCO spin cartridges (Amicon), and $0.2 \mu \mathrm{m}$ sterile filtered. mRNA production and sequence optimization was performed as previously described ${ }^{32,59}$. gRNA was procured from Synthego (sRGN Alb-T1), Avecia (SpyCas9 Alb-T1), and Agilent (modified sRGN Alb-T1 constructs). LNPs were characterized by dynamic light scattering (DLS) using a Wyatt Nanostar, Ribogreen (Thermo Fisher Scientific), for endotoxin (Endosafe, Charles River), by UPLC (Thermo Fisher Scientific), and cryoTEM (MIT) assays.

Animals. This study complied with all applicable sections of the Final Rules of the Animal Welfare Act regulations (Code of Federal Regulations, Title 9), the Public Health Service Policy on Humane Care and Use of Laboratory Animals from the Office of Laboratory Animal Welfare, and the Guide for the Care and Use of Laboratory Animals from the National Research Council. The protocol and any amendments or procedures involving the care or use of animals in this study was reviewed and approved by the Testing Facility Institutional Animal Care and Use Committee before the initiation of such procedures. Testing Facility Institutional Animal Care and Use Committee: Charles River Laboratories, Mattawan, Michigan 49071 (Macaca fascicularis) and Mispro Biotech Services, Inc. (C57BL/6j). C57BL/ 6j mice, age: 6 to 8 weeks old, sex: male, source: bred in captivity at Jackson Laboratories, Bar Harbor, Maine, Origin: Jackson Laboratories, Bar Harbor, Maine. Macaca fascicularis, age: $2-4$ years old, weight: $2.5-5.0 \mathrm{~kg}$, sex: male, source: bred in captivity at World Wide Primates, Inc., Origin: Mainland Asia.

Mouse studies to assess liver editing by LNPs. Animals were housed on a 12:12 $\mathrm{h}$ light/dark cycle at an ambient mean temperature of $23^{\circ} \mathrm{C}$ and humidity at $50 \%$. Experimental procedures were approved by the Institutional Animal Care and Use Committee (IACUC) review board. C57BL/6 animals (6-8 week-old male mice) were obtained from Jackson Laboratories (Bar Harbor, ME). For all mouse studies, animals were injected with a single intravenous dose of LNP-formulated mRNA and sgRNA through the tail vein, and after $96 \mathrm{~h} \pm 5 \%$ mice were euthanized for genome-editing analysis. Whole liver was flash-frozen in liquid nitrogen, homogenized and genomic DNA was extracted using the DNeasy Blood \& Tissue Kit (Qiagen) according to the manufacturer's instructions. A fragment containing the editing region was amplified using PCR and used for TIDE analysis.

\section{Subretinal injection of AAV5 vectors and analysis of genome editing in} photoreceptors of nonhuman primates. The Photoreceptor-specific GRK1 promoter was chosen to limit Cas 9 expression to photoreceptors ${ }^{60,61}$. A U6 promoterdriven T428 surrogate sgRNA, and the sRGN3.1 gene under GRK1 promoter were inserted between AAV2 ITRs and packaged in AAV5 capsids. The AAV5 vector was diluted to $1 \times 10^{12}$ or $2 \times 10^{12} \mathrm{vg} / \mathrm{mL}$ with phosphate-buffered saline containing $0.001 \% \mathrm{~F} 68$. The animals were anesthetized, and the eyes prepared for insertion of two 25 -gauge scleral ports $3 \mathrm{~mm}$ posterior to the limbus. An endoilluminator probe was inserted in one port and a subretinal injection cannula was inserted through the second port. The subretinal injection cannula was advanced to mid-vitreous and the small diameter injection cannula was advanced until it contacted the retinal surface. AAV5 vector solution of volume $0.1 \mathrm{~mL}$ was slowly delivered to induce a subretinal bleb. Once the dose was delivered, the injection cannula and endoilluminator were removed, the scleral ports were removed, and the sclerotomies were sealed using electrocautery.
Retinal punch biopsy. Following enucleation, the anterior chamber, lens, and vitreous humor from each eye were removed. Four radial cuts were made in the eyecup to flatten the globe. The subretinal bleb and a portion of the neurosensory retina distal to the bleb were collected with a 6-8 $\mathrm{mm}$ biopsy punch. The samples were snap-frozen on dry ice. Frozen retinal samples were pulverized using a Geno/ Grinder 2010 (SPEX SamplePrep, LLC) at 1500 revolutions per minute for $2 \mathrm{~min}$. The homogenized tissue was suspended in phosphate-buffered saline (PBS) and split into three tubes. Genomic DNA was extracted from approximately one-third of the retinal samples using QIAamp DNA Mini Kit (Qiagen), and indels were quantified by NGS as described above.

Statistical analysis and curve-plotting were conducted using GraphPad Prism 8. Where applicable, normal distribution was determined before suitable downstream statistical analysis using D'Agostino \& Pearson test. Unless stated otherwise, ${ }^{*} p<$ $0.05,{ }^{* *} p<0.01,{ }^{* * *} p<0.001$.

Patents. WO2019183150 covers sRGN3.1, SpaCas9, ShyCas9, and SmiCas9 sequences. Status: pending. Applicant: CRISPR Therapeutics AG, Bayer Healthcare LLC. Inventors: COHNEN, André, SCHMIDT, Moritz J., COCO, Wayne M., GAMALINDA, Michael B., GUPTA, Ashish, PITZLER, Christian, RICHTER, Florian, TEBBE, Jan, CHENG, Christopher J., TAKEUCHI, Ryo, REISS, Caroline W.

WO2019118935 covers SluCas9. Status: pending. Applicant: CRISPR Therapeutics AG, Bayer Healthcare LLC. Inventors: COHNEN, André, SCHMIDT, Moritz J., COCO, Wayne M., GUPTA, Ashish, TEBBE, Jan, SCHULENBURG, Cindy, PITZLER, Christian, GAMALINDA, Michael B. JACH, Sabine, RICHTER, Florian, ARUMUGHAN, Anup, SAALWÄCHTER, Corinna.

Reporting Summary. Further information on research design is available in the Nature Research Reporting Summary linked to this article.

\section{Data availability}

All data generated or analyzed during this study are included in this published article and its supplementary information files and are available from the corresponding author upon reasonable request. Parental Staphylococcus can be accessed on Uniprot. S. hyicus (Shy) GB: CP008747.1, Uniprot: A0A418JLD8, S. lugdunensis (Slu) NCBI reference sequence: NZ_GL622352.1, Uniprot: A0A133QCR3, S. microti (Smi) GB: JXWY01000132.1, Uniprot: A0A0D6XNZ8 and S. pasteuri (Spa) GB: CP004014.1. Sequences of engineered proteins used herein are available in Supplementary Table 2. NGS data of this study have been deposited in the Sequence Read Archive (SRA) at NCBI with the project number PRJNA731307. Source data are provided with this paper.

\section{Code availability}

All published algorithms used in this study are referenced in the Methods section. Custom-made algorithms are available from the corresponding author upon request. Supplementary Software is available with this manuscript.

Received: 15 January 2021; Accepted: 14 June 2021; Published online: 09 July 2021

\section{References}

1. Charpentier, E. \& Doudna, J. A. Rewriting a genome. Nature 495, 50-51 (2013).

2. Makarova, K. S. et al. An updated evolutionary classification of CRISPR-Cas systems. Nat. Rev. Microbiol 13, 722-736 (2015).

3. Jiang, F. \& Doudna, J. A. CRISPR-Cas9 structures and mechanisms. Annu. Rev. Biophys. 46, 505-529 (2017).

4. Makarova, K. S. et al. Evolutionary classification of CRISPR-Cas systems: a burst of class 2 and derived variants. Nat. Rev. Microbiol 18, 67-83 (2020).

5. Jinek, M. et al. A programmable dual-RNA-guided DNA endonuclease in adaptive bacterial immunity. Science 337, 816-821 (2012).

6. Mali, P. et al. RNA-guided human genome engineering via Cas9. Science 339, 823-826 (2013).

7. Ran, F. A. et al. Genome engineering using the CRISPR-Cas9 system. Nat. Protoc. 8, 2281-2308 (2013).

8. Hirakawa, M. P., Krishnakumar, R., Timlin, J. A., Carney, J. P. \& Butler, K. S. Gene editing and CRISPR in the clinic: current and future perspectives. Biosci Rep 40, BSR20200127 (2020).

9. Naso, M. F., Tomkowicz, B., Perry, W. L. \& Strohl, W. R. Adeno-associated virus (AAV) as a vector for gene therapy. BioDrugs 31, 317-334 (2017).

10. Gilbert, L. A. et al. CRISPR-mediated modular RNA-guided regulation of transcription in Eukaryotes. Cell 154, 442-451 (2013).

11. Chavez, A. et al. Highly efficient Cas9-mediated transcriptional programming Nat. Methods 12, 326-328 (2015). 
12. Komor, A. C., Kim, Y. B., Packer, M. S., Zuris, J. A. \& Liu, D. R. Programmable editing of a target base in genomic DNA without doublestranded DNA cleavage. Nature 533, 420-424 (2016).

13. Gaudelli, N. M. et al. Programmable base editing of $A \bullet T$ to $G \bullet C$ in genomic DNA without DNA cleavage. Nature 551, 464-471 (2017).

14. Anzalone, A. V. et al. Search-and-replace genome editing without doublestrand breaks or donor DNA. Nature 576, 149-157 (2019).

15. Pardi, N., Hogan, M. J., Porter, F. W. \& Weissman, D. mRNA vaccines-a new era in vaccinology. Nat. Rev. Drug Discov. 17, 261-279 (2018).

16. Ran, F. A. et al. In vivo genome editing using Staphylococcus aureus Cas9. Nature 520, 186-191 (2015).

17. Kim, E. et al. In vivo genome editing with a small Cas9 orthologue derived from Campylobacter jejuni. Nat. Commun. 8, 14500 (2017).

18. Adli, M. The CRISPR tool kit for genome editing and beyond. Nat. Commun. 9, 1911 (2018).

19. Keough, K. C. et al. AlleleAnalyzer: a tool for personalized and allele-specific sgRNA design. Genome Biol. 20, 167 (2019).

20. Streuli, M. et al. Target cell specificity of two species of human interferonalpha produced in Escherichia coli and of hybrid molecules derived from them. Proc. Natl Acad. Sci. USA 78, 2848-2852 (1981).

21. Crameri, A., Raillard, S.-A., Bermudez, E. \& Stemmer, W. P. C. DNA shuffling of a family of genes from diverse species accelerates directed evolution. Nature 391, 288-291 (1998).

22. Coco, W. M. et al. DNA shuffling method for generating highly recombined genes and evolved enzymes. Nat. Biotechnol. 19, 354-359 (2001).

23. Coco, W. M. et al. Growth factor engineering by degenerate homoduplex gene family recombination. Nat. Biotechnol. 20, 1246-1250 (2002).

24. van Overbeek, M. et al. DNA pepair profiling reveals nonrandom outcomes at Cas9-mediated breaks. Mol. Cell 63, 633-646 (2016).

25. Strecker, J. et al. Engineering of CRISPR-Cas12b for human genome editing. Nat. Commun. 10, 212 (2019).

26. Yourik, P., Fuchs, R. T., Mabuchi, M., Curcuru, J. L. \& Robb, G. B. Staphylococcus aureus Cas9 is a multiple-turnover enzyme. RNA 25, 35-44 (2019).

27. Hsu, P. D. et al. DNA targeting specificity of RNA-guided Cas9 nucleases. Nat. Biotechnol. 31, 827-832 (2013).

28. Tsai, S. Q. et al. GUIDE-seq enables genome-wide profiling of off-target cleavage by CRISPR-Cas nucleases. Nat. Biotechnol. 33, 187-197 (2015).

29. Fu, Y. et al. High-frequency off-target mutagenesis induced by CRISPR-Cas nucleases in human cells. Nat. Biotechnol. 31, 822-826 (2013).

30. Cradick, T. J., Qiu, P., Lee, C. M., Fine, E. J. \& Bao, G. COSMID: a web-based tool for identifying and validating CRISPR/Cas oOff-target sites. Mol. Ther. Nucleic Acids 3, e214 (2014).

31. Chen, J. S. et al. Enhanced proofreading governs CRISPR-Cas9 targeting accuracy. Nature 550, 407-410 (2017).

32. Vaidyanathan, S. et al. Uridine depletion and chemical modification increase Cas9 mRNA activity and reduce immunogenicity without HPLC purification. Mol. Ther. Nucleic Acids 12, 530-542 (2018).

33. Vaché, C. et al. Usher syndrome type 2 caused by activation of an USH2A pseudoexon: implications for diagnosis and therapy. Hum. Mutat. 33, 104-108 (2012).

34. Lino, C. A., Harper, J. C., Carney, J. P. \& Timlin, J. A. Delivering CRISPR: a review of the challenges and approaches. Drug Deliv. 25, 1234-1257 (2018).

35. Xu, C. L., Ruan, M. Z. C., Mahajan, V. B. \& Tsang, S. H. Viral delivery systems for CRISPR. Viruses 11, 28 (2019).

36. Liu, R. M. et al. Synthetic chimeric nucleases function for efficient genome editing. Nat. Commun. 10, 5524 (2019).

37. Hu, Z. et al. A compact Cas9 ortholog from Staphylococcus Auricularis (SauriCas9) expands the DNA targeting scope. PLoS Biol .18, e3000686 (2020).

38. Kleinstiver, B. P. et al. Broadening the targeting range of Staphylococcus aureus CRISPR-Cas9 by modifying PAM recognition. Nat. Biotechnol. 33, 1293-1298 (2015).

39. Saha, C. et al. Guide-free Cas 9 from pathogenic Campylobacter jejuni bacteria causes severe damage to DNA. Sci. Adv. 6, eaaz4849 (2020).

40. Edraki, A. et al. A compact, high-accuracy Cas9 with a dinucleotide PAM for in vivo genome editing. Mol. Cell 73, 714-726 (2019). e4.

41. Harrington, L. B. et al. Programmed DNA destruction by miniature CRISPRCas14 enzymes. Science 362, 839-842 (2018).

42. Pausch, P. et al. CRISPR-Cas $\Phi$ from huge phages is a hypercompact genome editor. Science 369, 333-337 (2020).

43. Kuscu, C., Arslan, S., Singh, R., Thorpe, J. \& Adli, M. Genome-wide analysis reveals characteristics of off-target sites bound by the Cas 9 endonuclease. Nat. Biotechnol. 32, 677-683 (2014).

44. Lin, Y. et al. CRISPR/Cas9 systems have off-target activity with insertions or deletions between target DNA and guide RNA sequences. Nucleic Acids Res. 42, 7473-7485 (2014).
45. Kosicki, M., Tomberg, K. \& Bradley, A. Repair of double-strand breaks induced by CRISPR-Cas9 leads to large deletions and complex rearrangements. Nat. Biotechnol. 36, 765-771 (2018).

46. Yang, H. et al. One-step generation of mice carrying reporter and conditional alleles by CRISPR/Cas-mediated genome engineering. Cell 154, 1370-1379 (2013).

47. Iyer, V. et al. Off-target mutations are rare in Cas9-modified mice. Nat. Methods 12, 479-479 (2015).

48. Iyer, V. et al. No unexpected CRISPR-Cas9 off-target activity revealed by trio sequencing of gene-edited mice. PLoS Genet. 14, e1007503 (2018).

49. Russell, S. et al. Efficacy and safety of voretigene neparvovec (AAV2hRPE65v2) in patients with RPE65-mediated inherited retinal dystrophy: a randomised, controlled, open-label, phase 3 trial. Lancet 390, 849-860 (2017).

50. Adams, D. et al. Patisiran, an RNAi therapeutic, for hereditary transthyretin amyloidosis. N. Engl. J. Med. 379, 11-21 (2018). https://doi.org/10.1056/ NEJMoa1716153.

51. Xue, H. Y., Guo, P., Wen, W.-C. \& Wong, H. L. Lipid-based nanocarriers for RNA delivery. Curr. Pharm. Des. 21, 3140-3147 (2015).

52. Beissert, T. et al. A trans-amplifying RNA vaccine strategy for induction of potent protective immunity. Mol. Ther. 28, 119-128 (2020).

53. Colella, P., Ronzitti, G. \& Mingozzi, F. Emerging issues in AAV-mediated in vivo gene therapy. Mol. Ther. Methods Clin. Dev. 8, 87-104 (2018).

54. Kleinstiver, B. P. et al. Engineered CRISPR-Cas9 nucleases with altered PAM specificities. Nature 523, 481-485 (2015).

55. Gong, S., Yu, H. H., Johnson, K. A. \& Taylor, D. W. DNA unwinding is the primary determinant of CRISPR-Cas9 activity. Cell Rep. 22, 359-371 (2018).

56. Certo, M. T. et al. Tracking genome engineering outcome at individual DNA breakpoints. Nat. Methods 8, 671-676 (2011).

57. Pinello, L. et al. Analyzing CRISPR genome-editing experiments with CRISPResso. Nat. Biotechnol. 34, 695-697 (2016).

58. Brinkman, E. K., Chen, T., Amendola, M. \& van Steensel, B. Easy quantitative assessment of genome editing by sequence trace decomposition. Nucleic Acids Res. 42, e168-e168 (2014).

59. Farelli, J. D. et al. Leveraging rational protein engineering to improve mRNA therapeutics. Nucleic Acid Ther. 28, 74-85 (2018)

60. Beltran, W. A. et al. rAAV2/5 gene-targeting to rods:dose-dependent efficiency and complications associated with different promoters. Gene Ther. 17, 1162-1174 (2010).

61. Maeder, M. L. et al. Development of a gene-editing approach to restore vision loss in Leber congenital amaurosis type 10. Nat. Med. 25, 229-233 (2019).

62. Nishimasu, H. et al. Crystal structure of Cas9 in complex with guide RNA and target DNA. Cell 156, 935-949 (2014).

63. Nishimasu, H. et al. Crystal structure of Staphylococcus aureus Cas9. Cell 162, 1113-1126 (2015)

\section{Acknowledgements}

The authors thank the following: Sabine Jach, Astrid Meyer, Helen Dietmar, Corinna Saalwächter, Katharina Schäfer, Viktoria Bazilyanska, Danuta Cichon and Anjanette Pickbrenner for molecular biology and assay support. Rike Wallbrecher for support with BFP disruption assays. Randi Mackler and Dominik Ziehe for protein purification and the execution and implementation of cell free assays. Keith Abe, Valerie Guerrero, Cornell Mallari, Luis Gamboa, and TeQuana Bayless for support with mouse experiments. Philipp Knyphausen, Andreas Neerincx, Gregory J. Cost, Alan Brooks, Karen Vo, Chad Cowan, Thomas J. Cradick, Tony Ho, John Kulman for thoughtful scientific discussions and suggestions. Hemangi Chaudhari, Soeren Turan, Eric G. Anderson and Brian Fochtman for critically reading the manuscript. Barbara Wan and Lisa Geller for alliance management and project support.

\section{Author contributions}

M.S., A.G., designed the research, performed experiments, analyzed the data, and wrote the manuscript. CG designed the research, performed the experiments, and analyzed data. R.T., M.L., A.N., C.P.W., M.A., A.K., A.B., and A.M.S. designed and performed experiments to select and test sgRNA in NHP and analyzed the data. K.W., C.R., S.S., K.K., and C.J.C. designed the research to test mRNA and sgRNA LNPs in mouse, performed experiments, and analyzed the data. C.J.C. contributed to writing the manuscript. S.G., C.B., C.P., B.C., M.G., and K.D. performed and analyzed the experiments. F.R. designed experiments and analyzed data. J.T., S.M., A.S., A.M.S., A.C., and W.M.C. designed and supervised the research and wrote the manuscript.

\section{Competing interests}

A.C., M.J.S., W.M.C., M.G., A.G., C.P., F.R., J.T., C.J.C., R.T., C.W.R., K.W., K.K., A.K., A.S., A.N., S.S. have filed patent applications through Bayer AG, Casebia LLC, and 
CRISPR Therapeutics on the results described herein. A.C., W.M.C., M.G., K.K., A.G., M.L., S.J.S., C.P., M.S., J.T. hold stock or shares in the affiliated companies.

\section{Additional information}

Supplementary information The online version contains supplementary material available at https://doi.org/10.1038/s41467-021-24454-5.

Correspondence and requests for materials should be addressed to A.C. or W.M.C.

Peer review information Nature Communications thanks the anonymous reviewer(s) for their contribution to the peer review of this work. Peer reviewer reports are available.

Reprints and permission information is available at http://www.nature.com/reprints

Publisher's note Springer Nature remains neutral with regard to jurisdictional claims in published maps and institutional affiliations. (c) (i) Open Access This article is licensed under a Creative Commons Attribution 4.0 International License, which permits use, sharing, adaptation, distribution and reproduction in any medium or format, as long as you give appropriate credit to the original author(s) and the source, provide a link to the Creative Commons license, and indicate if changes were made. The images or other third party material in this article are included in the article's Creative Commons license, unless indicated otherwise in a credit line to the material. If material is not included in the article's Creative Commons license and your intended use is not permitted by statutory regulation or exceeds the permitted use, you will need to obtain permission directly from the copyright holder. To view a copy of this license, visit http://creativecommons.org/ licenses/by/4.0/.

(C) The Author(s) 2021 